ISSN 2079-9292

www.mdpi.com/journal/electronics

Review

\title{
Organic Bioelectronic Tools for Biomedical Applications
}

\section{Susanne Löffler, Ben Libberton and Agneta Richter-Dahlfors *}

Swedish Medical Nanoscience Center, Department of Neuroscience, Karolinska Institutet, SE-171-77 Stockholm, Sweden; E-Mails: susanne.loffler@ki.se (S.L.); benjamin.libberton@ki.se (B.L.)

* Author to whom correspondence should be addressed; E-Mail: agneta.richter.dahlfors@ki.se; Tel.: +46-8-524-874-25; Fax: +46-8-34-26-51.

Academic Editors: Ruth Shinar and Emil J. W. List-Kratochvil

Received: 22 September 2015 / Accepted: 26 October 2015 / Published: 5 November 2015

\begin{abstract}
Organic bioelectronics forms the basis of conductive polymer tools with great potential for application in biomedical science and medicine. It is a rapidly growing field of both academic and industrial interest since conductive polymers bridge the gap between electronics and biology by being electronically and ionically conductive. This feature can be employed in numerous ways by choosing the right polyelectrolyte system and tuning its properties towards the intended application. This review highlights how active organic bioelectronic surfaces can be used to control cell attachment and release as well as to trigger cell signaling by means of electrical, chemical or mechanical actuation. Furthermore, we report on the unique properties of conductive polymers that make them outstanding materials for labeled or label-free biosensors. Techniques for electronically controlled ion transport in organic bioelectronic devices are introduced, and examples are provided to illustrate their use in self-regulated medical devices. Organic bioelectronics have great potential to become a primary platform in future bioelectronics. We therefore introduce current applications that will aid in the development of advanced in vitro systems for biomedical science and of automated systems for applications in neuroscience, cell biology and infection biology. Considering this broad spectrum of applications, organic bioelectronics could lead to timely detection of disease, and facilitate the use of remote and personalized medicine. As such, organic bioelectronics might contribute to efficient healthcare and reduced hospitalization times for patients.
\end{abstract}


Keywords: organic bioelectronics; nanomedicine; regenerative medicine; drug delivery; tissue engineering; tissue microbiology

\section{Introduction}

Over the last decade, organic bioelectronics have become more popular and widely used in biological research and medicine [1-4]. Organic bioelectronics are based on conductive polymers and inherit their flexibility, optical transparency and electrical and ionic conductivity. Further, they can be adapted and functionalized in a wide variety of ways thanks to organic chemistry methods. This combination of structural and functional flexibility makes them especially well-suited to applications in the medical field since it allows precise modeling of various attributes of cells and human tissues [5,6]. This review demonstrates the most recent developments in organic bioelectronics with important implications for biological research and medicine.

Conductive polymers are organic compounds featuring a conjugated backbone and doping agents, which can reversibly associate or dissociate depending on the redox state of the backbone. In the conjugated backbone, chemical bonds mesomerize into alternating single and double bonds. In this energetically favourable state electrons are localized in the fully occupied valence band represented by the "highest occupied molecule orbital" (HOMO) separated from the unoccupied conduction band, represented by the "lowest unoccupied molecule orbital" (LUMO). HOMO and LUMO are energetically separated by the band gap energy $\left(\mathrm{E}_{\mathrm{g}}\right)$. Thus, pristine conjugated polymers are semiconductors and $\mathrm{E}_{\mathrm{g}}$ determines their optical properties. Chemical tuning can be performed in order to adjust Eg. Generally, more planar polymer structures feature smaller band gap energies within the energy spectrum of visible light [7-14].

Doping is the introduction of additional charge carriers into the conjugated polymer and increases the electrical conductivity [10]. Thus, doped conjugated polymers are called conductive polymers. Doping occurs by chemical reduction (n-type) or oxidation (p-type). The dopant acts as counter-ion to equilibrate the charge along the oxidised or reduced backbone. Conducting polymers therefore act like ionic complexes and their properties are largely dependent on the conjugated backbone, redox state and doping agent [15]. In most organic bioelectronics applications, p-doped conductive polymers are used due to their stability towards oxidation.

In general, the polymeric nature of the conductive polymers confers structural flexibility. This means that a wide variety of methods such as electropolymerisation on any possible conducting substrate, bar coating, screen printing, inkjet printing, and vapor phase polymerization can be used for fabrication. Beyond allowing for simple fabrication, the structural flexibility of conducting polymer materials allows seamless integration into existing experimental setups commonly used in biomedical research. Their transparency and tunable optical properties make most conductive polymers conducive to various forms of microscopy.

Unique features like ionic conductivity follow from the redox properties of conductive polymers. Low redox potentials of conducting polymer backbones in aqueous electrolyte allow reversible oxidation and reduction of the material, which creates excess charges in the polymer matrix. Charge equilibration 
requires controlled ion movement into or out of the polymer and gives rise to ionic currents. Ionic conductivity allows the use of organic bioelectronics devices to translate electronic currents into ionic flow $[10,16,17]$. While ionic currents are a common feature of biological communication, they are difficult to control or mimic with contemporary methods. Electronic currents are, however, sufficiently easy to control. Thus, organic bioelectronics might provide the missing link between electronics and biology and might find extensive application in developing fields like brain-computer interfacing, but also give rise to emerging applications in bioelectronic interfaces.

In this review we will highlight a series of organic bioelectronic devices that function based on the principle of iontronics [18]. Furthermore, we will introduce a panel of active surfaces based on organic bioelectronics, which help to mimic dynamic biological environments and allow the modulation of cell behavior with high degrees of spatiotemporal control. Since self-controlled systems are the ultimate goal in any bioelectronic interface aiming to alleviate pathological states, sensing applications based on organic bioelectronics are introduced as well. Further, there will be a focus on specific applications of organic bioelectronics in neuroscience, infection research and cell biology.

\section{Electronically Controlled Ion Transport}

Ions are essential signaling entities in biological systems. However, methods are scarce that allow control of the number of ions delivered to a system. The established technique of ionotophoresis is based on electrophoretic ion transport, thus lending itself to drug delivery applications, such as transdermal administration of therapeutic agents [19-21]. However, precise spatio-temporal control is difficult to achieve in this system.

To overcome the shortcomings of iontophoresis, a series of organic bioelectronic devices translating electronic currents into precisely controlled ionic currents have been developed (Figure 1). Based on their common working principle, these devices are called organic electronic ion pumps (OEIPs). As a common feature, OEIPs are based on poly(3,4-ethylenedioxythiophene):poly(styrenesulfonate) (PEDOT:PSS), a chemically stable polymer-polyelectrolyte system with electronic and ionic conductivity. The PEDOT:PSS film is patterned onto a polyethyleneterephthalate (PET) substrate to create at least one source and one target electrode. The source and target electrodes are connected by a polymer channel (mostly overoxidized PEDOT:PSS), that is conductive for ions but not for electrons. The layout and size of the electrodes and channel is flexible and can be adapted to the specific application. On top of the PEDOT:PSS electrodes, a hydrophobic, insulating material is patterned (mostly SU8), so that liquid reservoirs are created over the source and target electrodes. The source reservoir is filled with an electrolyte containing a specific cation that is to be transported and the target reservoir is filled with an electrolyte, chosen to support the application. An electrical potential applied over the OEIP source $(+)$ and target $(-)$ electrodes, will oxidize $\left(\right.$ PEDOT $^{0} \rightarrow$ PEDOT $\left.^{+}+\mathrm{e}^{-}\right)$at the anode and reduce $\left(\mathrm{PEDOT}^{+}+\mathrm{e}^{-} \rightarrow \mathrm{PEDOT}^{0}\right.$ ) at the cathode. The negative charge of the polyelectrolyte $\left(\mathrm{PSS}^{-}\right)$ will be equilibrated with cations moving from the source electrolyte reservoir through the channel to the target electrolyte reservoir. 
a)

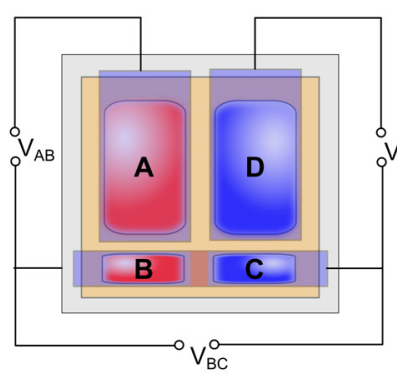

b)

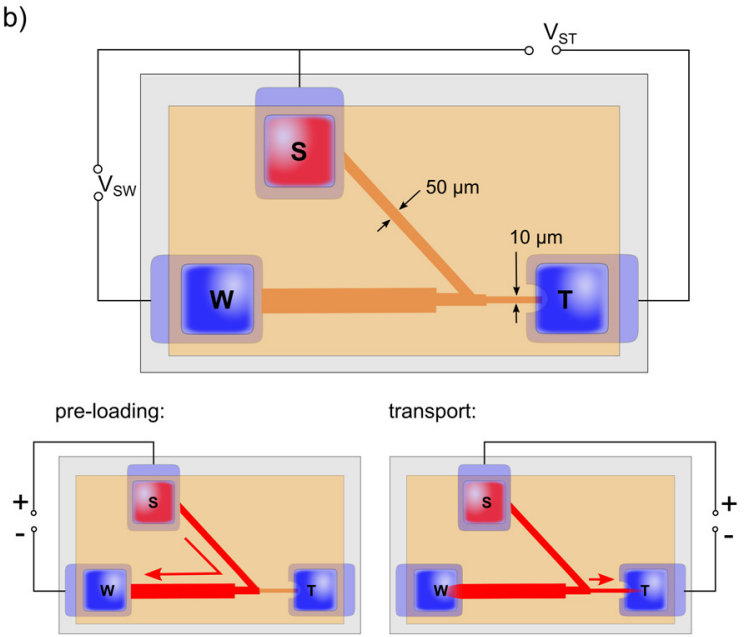

c)
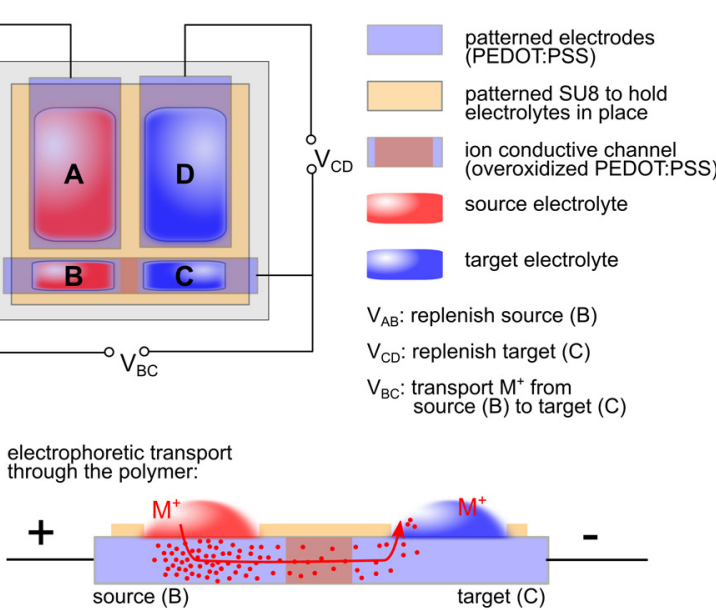

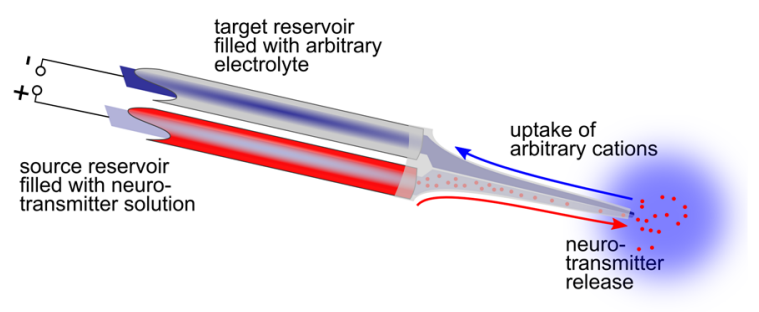

Figure 1. Electronically controlled ion transport using OEIPs. (a) Four PEDOT:PSS electrodes (A-D) are shown with electrolyte reservoirs held in place by a thin layer of SU8. The source $(\mathrm{B})$ and the target $(\mathrm{C})$ electrodes are connected by an overoxidised PEDOT:PSS channel. Two larger electrodes (A and D) are used to replenish the smaller electrodes (B and C); (b) The OEIP layout is changed to accommodate three PEDOT:PSS electrodes, source (S), waste (W) and target (T). A pre-loading circuit is created to enable tight spatiotemporal control over the transport. For pre-loading the channel, source (S) and waste (W) electrodes are adressed. To rapidly initiate transport, source $(\mathrm{S})$ and target $(\mathrm{T})$ electrodes are addressed as shown in the transport circuit; (c) Syringe like OEIP suitable for implantation and in vivo experimentation. By addressing source and target electrodes immersed in the respective reservoirs, biomolecules are released from the tip of the device, which can be placed in direct contact with specific biological tissue or cell type.

The first device of a series of OEIPs was developed to control $\mathrm{Ca}^{2+}$ signalling in neuronal cells by electronic control of ion transport through the channel of the OEIP (Figure 1a) [22]. Here, four PEDOT:PSS electrodes were patterned. A layer of SU8 was added to hold electrolyte reservoirs on top of the electrodes (Figure 1a, A-D). An overoxidated PEDOT:PSS channel was created between the source (Figure 1a, B) and target (Figure 1a, C) electrode. Since the capacity of the OEIP is defined by the number of redox sites, and since PEDOT:PSS in its pristine state is more oxidized than reduced, the amount of redox sites at the source electrode is the limiting factor of the OEIPs ion transport capability. Therefore, the source and target electrodes were integrated in an individual electrical circuit with another, larger electrode (Figure 1a, A and D) so that the source electrode (Figure 1a, B) can be replenished until the capacity of the larger electrode (Figure 1a, A) is reached. 
Initially, ion transport was investigated for the monovalent cation $\mathrm{K}^{+}$and the divalent cation $\mathrm{Ca}^{2+}$. The cations were applied on the source electrode (Figure 1a, A and B) in form of $10 \mathrm{mM}$ electrolytes of the chloride salt. Electrolytes $(10 \mathrm{mM})$ of the acetate salt (calcium acetate when $\mathrm{K}^{+}$was transported, potassium acetate when $\mathrm{Ca}^{2+}$ was transported) were applied to the target electrode (Figure 1a, C and D). Potentials between 5 and $10 \mathrm{~V}$ were used to transport $\mathrm{K}^{+}$or $\mathrm{Ca}^{2+}$, and the transport charge was determined from the time integral of the transport current. Thus, it was found that about $150 \mathrm{nmol} \mathrm{K}$ were transported with $15 \mathrm{mC}$ and $30 \mathrm{mC}$ transport charge was required to transport the same amount of $\mathrm{Ca}^{2+}$. Thus, transport efficiencies per ion charge close to $100 \%$ were established for $\mathrm{K}^{+}$and $\mathrm{Ca}^{2+}$. Comparing the amount of ions in the target reservoir after active transport and passive diffusion, the ON/OFF ratio of the device was established to be 300:1 [22]. When protons $\left(\mathrm{H}^{+}\right)$were transported using the same device, transport efficiencies were found to be close to $100 \%$ (one proton per electron) with an ON/OFF ratio of $>1000: 1$ [23].

Since the transport via the OEIP does not mechanically disturb the sample, it was possible to use the device to generate delicate $\mathrm{pH}$ gradients and proton oscillations with tight electronic control [23]. Varying the ON time of the OEIP can generate $\mathrm{pH}$ gradients bridging three orders of magnitude $\left[\mathrm{H}^{+}\right]$ over a distance of $5 \mathrm{~mm}$ in the target electrolyte. Addressing the device with 10-15 s monopolar, rectangular pulses with 5 or $10 \mathrm{~V}$ amplitude every 4 min yielded $\mathrm{pH}$ oscillations in the medium with a periodicity of $5 \mathrm{~min}$, which is a time scale observed in many cell signalling events, such as ion controlled gene transcription. Thus, the potential of the OEIP to precisely control and modulate cellular signalling events was recognized. However, the design of the device with wide channels of $50 \mu \mathrm{m}-4 \mathrm{~mm}$ led to delayed delivery times due to residual ions in the material. Thus, cellular responses were delayed and electronic control could not be exerted as accurately as possible.

To translate electronic currents into precise chemical cues controlling fast neuronal signalling, the OEIP device layout was adapted to $(i)$ expand the transport repertoire from metal cations $\left(\mathrm{H}^{+}, \mathrm{K}^{+}, \mathrm{Ca}^{2+}\right)$ to biomolecules; (ii) provide increased spatiotemporal control with channel outlets around the size of a single neuronal cell; and (iii) maximise temporal control over the delivery and reduce delivery delay times from minutes to seconds [24]. This was achieved by the next generation of OEIP devices (Figure 1b). The 10- $\mu \mathrm{m}$ OEIP featured a $10 \mu \mathrm{m}$ channel outlet and a pre-loading circuit with which the channel can be loaded with biomolecules before delivery to the target electrode (Figure 1b, pre-loading). Through the pre-loading circuit, a waste electrode is introduced alongside source and target electrodes. By addressing the source and waste electrodes with $20 \mathrm{~V}$ for $3 \mathrm{~min}$, the channel was pre-loaded with biomolecules to be delivered. Transport of the biomolecule to the target electrode was initiated by applying a potential of $20 \mathrm{~V}$ between source and target electrode (Figure 1b, transport). Using the preloading circuit, the delay in transport was reduced to a few seconds. When the neurotransmitter acetylcholine (ACh) was transported as a candidate for a biologically active compound, delivery rates of $0.26 \pm 0.02 \mathrm{pmol} \mathrm{s}^{-1}$ and $0.55 \pm 0.03 \mathrm{pmol} \mathrm{s}^{-1}$ were established when transport was driven with 20 and $40 \mathrm{~V}$. Transport efficiencies of of $103 \pm 3 \%$ and an ON/OFF ratio of about 400:1 was established for transport of the neurotransmitter ACh [24].

Assessment of the retention of biological activity of the transported biomolecule was examined by using neuron-like cells as biosensor for the delivered neurotransmitter ACh. Human neuroblastoma cells, known to express ACh receptors (AChR) were cultured on the target electrode and cell culture medium was used as electrolyte in the target reservoir. By addressing the device and initiating the transport of 
ACh through the channel to the neuroblastoma cells on the target electrode, it was found that cells responded by an intracellular $\mathrm{Ca}^{2+}$ response mediated by AChR-binding. Since the $10 \mu \mathrm{m}$ OEIP can be operated with tight spatiotemporal control, the range of cellular control was established by monitoring the intracellular $\mathrm{Ca}^{2+}$ response to ACh transport at 50 and $150 \mu \mathrm{m}$ from the outlet. A neuronal cell, growing in a radius of $50 \mu \mathrm{m}$ around the outlet point responded with an increase in intracellular $\left[\mathrm{Ca}^{2+}\right.$ ] of about $30 \%$ within a time of half maximal response $t_{\max 1 / 2}=11 \mathrm{sec}$. Another cell, growing within a $150 \mu \mathrm{m}$ radius from the outlet responded significantly more slowly and with negligible signal amplitude. Thus, it was found that single cell control could be established using the $10 \mu \mathrm{m}$ OEIP [24].

Due to the tight spatiotemporal control of ACh transport, it was not only possible to excite single cells, but also to achieve repeated stimulation of neuron like cells using ACh. Since the high [ACh] at the outlet is maintained locally, it is expected to instantly collapse due to rapid diffusion when the transport is interrupted. When $0.2-2 \mathrm{~s}$ pulses of $\mathrm{ACh}$ transport were applied in a regular pattern, oscillations of intracellular $\mathrm{Ca}^{2+}$ were observed. When the ACh transport was stopped, the high local [ACh] diffused in the bulk medium and the cellular response subsided. Thus, it was possible to re-excite cells with specific patterns of stimuli whose frequency was shown to control the frequency of $\mathrm{Ca}^{2+}$ oscillations in the stimulated cells and whose duration was shown to control the amplitude of the cellular response [24].

The direction indicated by the tightly controlled $10 \mu \mathrm{m}$ OEIP is to create a multitude of individually addressable delivery channels for chemical cues to gain dynamic control of cellular microenvironments. This can be achieved by introducing transistor functionality similar to the addressing schemes found in active matrix displays to create active chemical circuits in high-resolution patterns. The ion bipolar junction transistor (IBJT), which is an electrophoretic, chemical transistor, was developed to enable the construction of more advanced delivery schemes controlling the flow of ions and charged biomolecules [25-27]. The IBJT consists of three channels (emitter, collector and base). The terminals, where reservoirs for electrolytes are defined, are all made of conductive PEDOT:PSS. Emitter and collector channels consist of over-oxidized PEDOT:PSS. The base channel is made of an anion selective membrane. The three channels meet in a junction where they interface a neutral cross-linked gel layer. Transport of cations from the emitter to the collector requires that voltage is applied across the emitter-collector $\left(V_{\mathrm{EC}}>0 \mathrm{~V}\right)$ and that the junction is conductive. This is obtained by varying the salt concentration in the cross-linked gel layer. In the active mode $\left(V_{\mathrm{EB}}>0 \mathrm{~V}\right)$, chloride ions $\left(\mathrm{Cl}^{-}\right)$migrate through the base into the junction where they get compensated by cations from the emitter. This increases the ionic conductivity between the emitter and collector allowing the molecules that are to be delivered from the emitter to the collector, passing through the gel [26]. Hence, the ionic current between the emitter and collector, i.e., the amount of delivered molecules, becomes a function of the potential applied to the base. The functionality of the IBJT was demonstrated using real-time $\mathrm{Ca}^{2+}$ imaging recordings of adherent neuroblastoma cells cultured by the outlet on the collector electrode. Keeping the IBJT in the OFF state $\left(\mathrm{V}_{\mathrm{EC}}=10 \mathrm{~V}\right.$ and $\left.\mathrm{V}_{\mathrm{EB}}=-1 \mathrm{~V}\right)$, baselines of the intracellular $\left[\mathrm{Ca}^{2+}\right]$ were recorded. The device was then switched to its active mode by reversing the bias voltage of the base $\mathrm{VEB}$. $\mathrm{As}^{-} \mathrm{Cl}^{-}$migrates through the base channel into the junction, migration of ACh from emitter to collector is achieved. Local delivery of ACh was initiated by applying $\mathrm{VEB}_{\mathrm{EB}}=4 \mathrm{~V}$ to the IBJT. As cells located at the collector outlet were exposed to ACh, an immediate increase of intracellular $\mathrm{Ca}^{2+}$ was observed. When the IBJT was switched to its OFF state, ACh delivery was terminated. As a consequence, the intracellular $\left[\mathrm{Ca}^{2+}\right]$ declined and returned to a basal level. Re-exposure 
to ACh by applying $\mathrm{VEB}=4 \mathrm{~V}$ led to initiation of a renewed $\mathrm{Ca}^{2+}$ response and showed that cells were able to be re-excited. Thus, transistor functionality and transistor mediated control of neuronal activation was demonstrated using a chemical transistor [26]. Gaining electronic control of chemical circuits as demonstrated by the IBJT is a crucial step to establish dynamically controlled microenvironments. Considering the variety of applications in modern laboratories, the chemical transistor has the potential to initiate a new era of automation in wet lab technology, much like the electronic transistor did in electrical engineering.

While the properties of the OEIP and its related technology are favorable for in vitro applications, tight spatiotemporal control of transport without initial burst release and the absence of liquid flow are essential to establish a delivery application for neuroactive substances in vivo. Since the original flat design of the OEIP is not suitable for intracerebral application, the OEIP was tailored into a flexible syringe-like shape for surgical implantation (Figure 1c) [28]. The planar device was separated into two encapsulated electrode-electrolyte systems, the source and target compartments, enclosing anode and cathode. The source compartment is filled with the substance to be delivered and the cathode system with an electrolyte. By cutting the over-oxidized and electronically insulated ion channel in half, the tip from the source and target electrodes comes into direct contact with the target system. As demonstrated earlier [23,24], the target electrolyte can be complex cell media or buffer and can thus be any type of extra- or intracellular fluid when used in vivo. To complete the electrochemical circuit, cations are extracted from the target system into the OEIP target compartment. Since this process is not specific, the vast pool of extracellular ions will readily replenish the target system.

The syringe-like OEIP (Figure 1c) was used to demonstrate transport of common neurotransmitters like $\gamma$-aminobutyric acid (GABA) and glutamic acid (Glu) as well as aspartic acid (Asp). Importantly, the transported neurotransmitters maintained their biological activity. Primary cultures of astrocytes, known to express Glu receptors, were stimulated with OEIP transported Glu [28]. As the working principle is the same as in the planar devices, the voltage drop occurs primarily across the channel, resulting in negligible electric fields in the target system. Hence, the cells are not stimulated by the applied potential or electric field, but by the neurotransmitter released from the device. When transported Glu reaches the tip of the device, it is released into the target electrolyte binding to Glu receptors (GluR) on the membrane of adjacent cells. GluR activation results in an influx of $\mathrm{Ca}^{2+}$ into the cell, which is recorded by intracellular $\mathrm{Ca}^{2+}$ imaging.

To demonstrate the in vivo application of the device, the syringe-like OEIP was used to modulate mammalian sensory function by achieving non-invasive delivery of Glu to the perilymph in the cochlea of guinea pigs. The tip of the syringe-like OEIP was mounted at the bone beside the cochlea round window membrane (RWM). Using the RWM as a port of diffusive entry into the cochlea, continuous delivery of Glu was achieved at a constant voltage for $1 \mathrm{~h}$. By monitoring the brain's ability to perceive sound of specific frequencies, the so-called auditory brainstem responses (ABR), hearing sensitivity was assessed before and after Glu delivery. During the $1 \mathrm{~h}$ exposure, ABR measurements showed that the animal suffered from significant loss of higher frequencies at the base where Glu enters via the RWM, whereas the control group exposed to transported $\mathrm{H}^{+}$showed no change in hearing sensitivity. In addition to confirming the excitotoxic effect of Glu, this experiment demonstrated the inert nature of the OEIP in vivo delivery system, meaning that the device application and molecular transport itself was not damaging. It was successfully demonstrated that the syringe-like OEIP can relay electronic signals into 
chemical cues that influence neuronal signaling. Thus, this was the first proof of concept to demonstrate the OEIP technology's potential for machine-to-brain interfaces.

Alternative concepts for electronically controlled ion transport are based on mesoporous carbon membranes [29] and TiO2 nanochannels [30]. With dimensions smaller than the Debye length of the electric double layer, direct manipulation of ions in the nanochannels becomes possible via the surface charge or an electrical field. It was shown that diffusion of charged dye molecules, and small ions $\left(\mathrm{K}^{+}, \mathrm{Cl}^{-}\right)$ through a mesoporous carbon membrane can be controlled by an electrochemical potential, whereas the electric field controlled transport of $\mathrm{Mg}^{2+}$ and $\mathrm{Na}^{+}$in $\mathrm{TiO}_{2}$ nanochannels.

\section{Self-Controlled Biomimetic Systems}

Closed-loop systems, able to sense a pathological state and exert some kind of stimulation in order to balance the environment, are gaining increasing attention, primarily in applications related to neuroscience [31,32]. Miniaturized, low-power brain-computer interface microsystems enable high performance on-line signal processing and an array of chronically implantable, biocompatible and stable electrodes can be used as biointerfaces in these advanced neuronal closed-loop systems [31]. Cortical brain computer interfaces, used to convey precise directions of movement [33,34], show great promise for development of robotic prostheses $[35,36]$. While the technology has improved significantly over the last years, self-controlled systems using organic bioelectronics are still rare.

The unique property of the OEIP to transform electrical signals into release of chemical substances is similar to the function of a nerve cell in which electrical signals, travelling along the axon in form of action potentials, are transformed into the release of neurotransmitters at the axon terminals. The value of the OEIP technology in machine-to-brain interface applications has been described in detail in an earlier review [2]. Building on that notion, the OEIP technology was used to fabricate an organic electronic biomimetic neuron (OEBN) to enable auto-regulated neuromodulation (Figure 2). In its most simplified form, a neuron can be considered a chemical-to-electrical-to-chemical signal transduction. Whereas information over long distances is conveyed by electrical signals, chemical signals function as input and output at both ends of the nerve cell to provide biochemical specificity. Chemical sensing is mediated by receptors on the cell body. Binding of a neurotransmitter to its receptor leads to slight depolarization or repolarization of the neuronal membrane. Integration of the chemical inputs eventually leads to generation of an action potential. This electrical signal is quickly transmitted along the axon. At the axon terminals, the action potential is again converted into a chemical signal by initiating fusion of neurotransmitter-containing vesicles with the neuronal membrane and thus release of neurotransmitters from the axon terminals. 


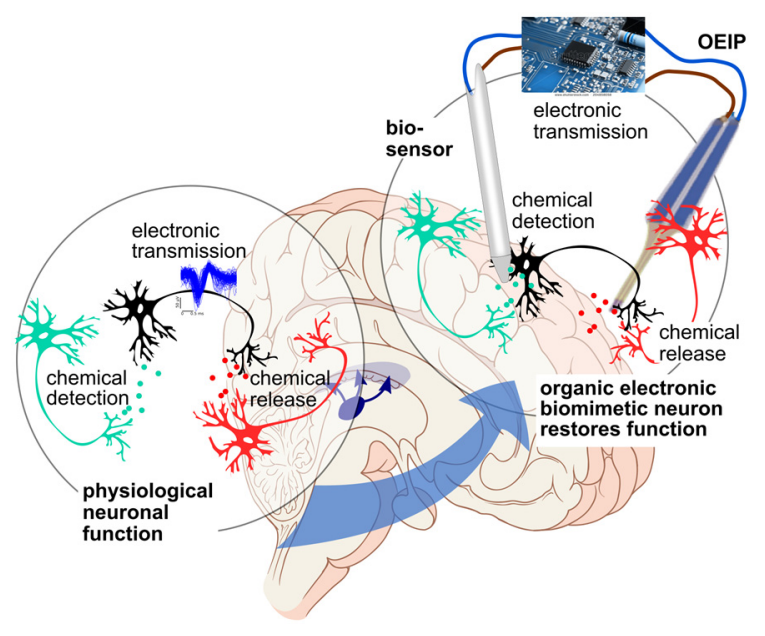

Figure 2. Organic electronic biomimetic neuron (OEBN). A normal physiological neuronal function is characterised by chemical-to-electrical-to-chemical signalling. A neuron senses a chemical signal which is converted to an electronic action potential that travels down the neuron. The action potential eventually triggers the release of neurotransmitters at the axon terminal. The OEBN mimics the chemical-to-electrical-to-chemical signalling of neurons. A biosensor detects a change in neurotransmitter concentration, which sends an electrical signal to the syringe OEIP and triggers the release of neurotransmitters.

The OEBN was built to mimic this fundamental aspect of neuronal function (Figure 2) [37]. Chemical-to-electrical signal transduction is achieved by a selective biosensor. Commercial amperometric biosensors utilize enzymatic reactions to oxidize or reduce the chemical compound to be detected. This leads to an electronic sensor current, which is directly proportional to the concentration of the chemical to be detected. For the OEBN, amperometric Glu and ACh sensors were utilized. When operated with $0.6 \mathrm{~V}$ and $0.5 \mathrm{~V}$ against a built-in $\mathrm{Ag} / \mathrm{AgCl}$ reference electrode, respectively, the highly selective biosensor yielded two electrons for each Glu and four electrons for each ACh. To create an automated control circuit, the sensor current was used as an input signal for the OEIP control software. In accordance with neuronal excitation being dependent on specific threshold activation, neurotransmitter transport through the OEIP, and thus electrical-to-chemical transition, was initiated when an arbitrary threshold of $80 \mu \mathrm{M}$ Glu was reached at the sensor. Now, if [Glu] exceeds $80 \mu \mathrm{M}$ in a cell culture dish in which the sensor part of the OEBN is located, the OEIP part of the OEBN is activated in a remote cell culture and neurotransmitter transport is initiated. Based on the essential principles of neuronal chemical-to-electrical-to-chemical signal translation during neuronal transmission, two remote neuronal populations were interfaced using the OEBN. These initial experiments show the potential of the OEBN technology to be able to support or replace damaged neuronal connections in the brain (Figure 2). This holds great potential for application in neurodegenerative diseases like Alzheimer's or Parkinson's syndrome. Since wireless transmission of the electrical signal is possible [38], application in paralyzed patients is potentially possible with ACh release at the motor end plate, which can control muscle contraction and thus help to regain the ability to move while natural muscular innervation might be lost.

In another approach, a sensing-coupled drug delivery system was developed based on the polymer polypyrrole (PPy) doped with the anionic drug adenosine triphosphate (ATP). A self-regulated system 
was achieved based on ATP release occurring in the presence of chemical triggers, such as reducing agents (e.g., hydrazine), the bioreductant dithiothreitol and changes in the local $\mathrm{pH}$ [39].

\section{Organic Bioelectronic Active Surfaces}

The surface of a material creates an interface between the material and its environment. For a given application, surfaces with specific properties can be developed. A prominent advantage of organic bioelectronics is that modulating their redox state leads to changes in the surface properties. Accordingly, it has been shown that cells distinguish between oxidized and reduced surfaces of organic bioelectronic materials. Furthermore, depending on the material, redox switching the surface can induce cell stimulation, attachment, differentiation, and morphology changes [40-42]. Thus, organic bioelectronics can be used to model different dynamic states often encountered in biological systems. Using organic bioelectronics as active surfaces to induce certain behavior in cells is advancing the field of cell biology by providing biomimetic surfaces that allow the study of cell behavior close to in vivo behavior [43]. A panel of active surfaces has been developed using organic bioelectronics in recent years and used to guide cell behavior upon an electronic trigger (Figures 3 and 4).

a)

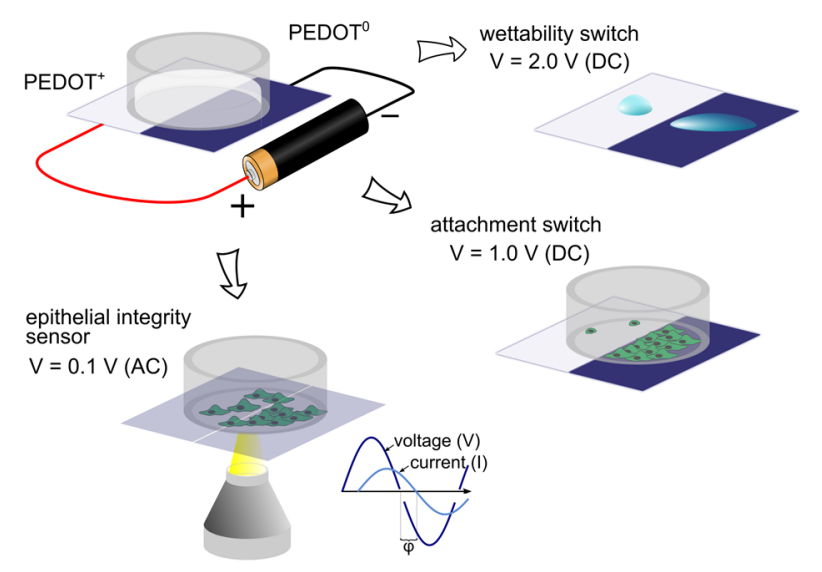

b)

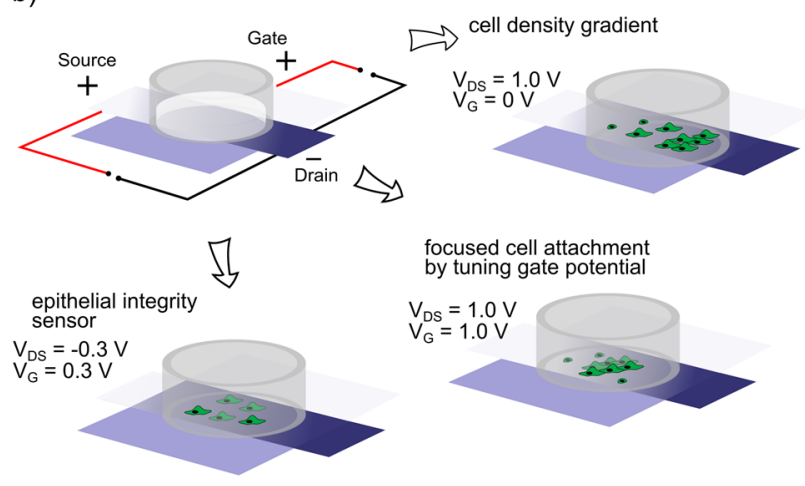

Figure 3. Organic bioelectronics used for electrochemical control of surface energy. (a) A two-electrode surface switch is used to display oxidized or reduced surfaces upon electrochemical switching. Using the two-electrode switch, cell attachment can be controlled, since cells tend to grow on the reduced surface but not on the oxidized surface. Also, applying an alternating sinusoidal potential, a transparent two-electrode sensor can be created by monitoring the phase delay of the measured current. This sensor can be used to monitor epithelial barrier formation and disruption. Simultaneous optical and electronic measurements can be performed as the sensor is fully transparent; (b) An organic electrochemical transistor (OECT) is created by adding a gate electrode to a conductive channel between source and drain electrodes. Applying a constant potential over the channel, cell density gradients can be created due to the cells preference for a certain surface redox state. Tuning the gate potential, cellular attachment can be focused to a narrow area along the channel of the OECT. By pulsing the gate potential and measuring current transients, the conductivity of the channel can be determined. Thus, the OECT can be applied as sensor for epithelial integrity as well. 
a)

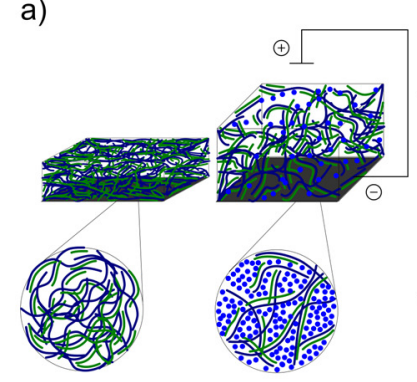

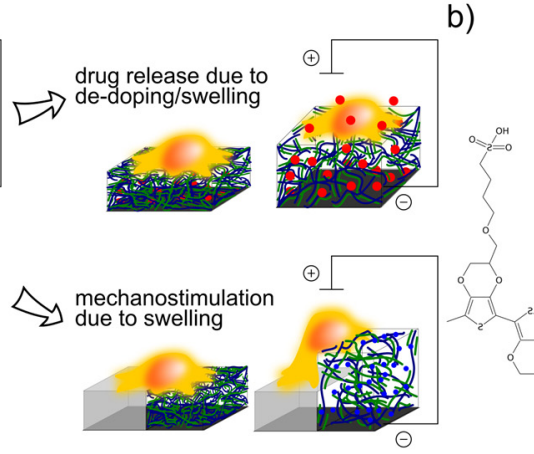

b)

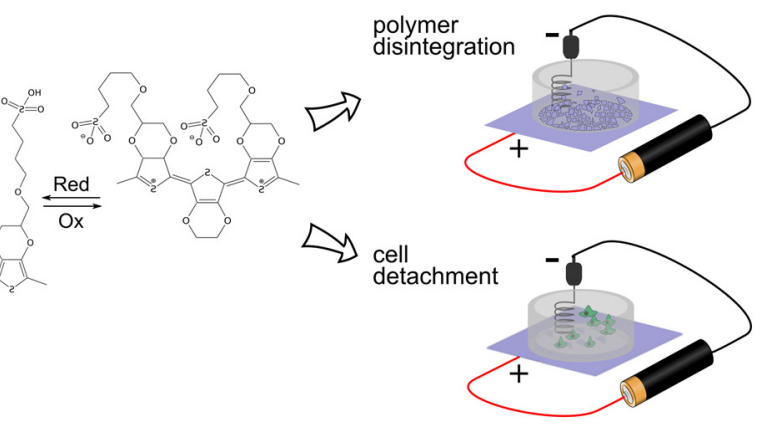

Figure 4. Organic bioelectronics using electrochmical swelling and de-doping properties of conductive polymers. (a) Swelling behavior in conducting polymers depends on the incroporation of solvent molecules into the polymer. Diffusion of solvent into the polymer is triggered by electrochemical switching and the creation of excess charges in the polymer matrix. Electrochemical reduction of p-doped conducting polymers leads to dissociation of the polymer and doping ion. The de-doping/swelling properties of conducting polymers can be used in organic bioelectronic devices for electronically triggered drug release and facilitates mechanostimulation of cells; (b) Self-doped conducting polymers have been developed that undergo immense structural changes upon electrochemical switching. The self-doped conducting polymers PEDOT-S:H has been developed to disintegrate upon oxidation when immersed into an electrolyte. Using the same polymer, electronically triggered release of cells from their substrate has been achieved.

Electrochemical reduction or oxidization of a conducting polymer leads to alteration of charges at the polymer backbone. Depending on the backbone charge, doping ions are tightly associated or dissociated with the backbone [10]. Thus, the chemical properties of the doping agents are determinants of the surface properties during reversible redox switching of the conducting polymer.

To create wettability switches based on conducting polymers doping agents representing monomeric surfactants, such as dodecylbenzenesulfonate (DBS) or perfluorooctanesulfonate (PFOS) ions are most widely used, while multiple conjugated polymers such as polyaniline (PANI), polypyrrole (PPy) and poly(3-hexylthiophene) (P3HT) are employed as backbones [44-46]. While the micro- and nanostructure of the film play a role [46], the chemical properties of the dopant are the most influential in determining the wettability of a surface. In an oxidized state, the backbone carries positive charges and the hydrophilic group of the surfactant is associated with the polymer, while the hydrophobic tail is sticking out towards the surface, creating a hydrophobic film with large surface energy (Figure 3a, wettability switch). In a reduced state, the dopant dissociates and the hydrophilic group will be free to interact with water dipoles on the surface, creating a more hydrophilic surface [44].

Based on changes in surface energy, cell attachment switches have been developed that allow for attachment of cells in the reduced state, but not in the oxidized state (Figure 3a, attachment switch). It appears that an altered surface chemistry and charge cause a distortion of the conformation of fibronectin, a protein essential for cellular attachment [42,47]. Devices that allow control of the surface energy of a conducting polymer film can be simple two-electrode switches, presenting the fully oxidized and fully reduced state of the polymer on each single electrode, with a gradient of surface energy on the 
conducting polymer in between. By addressing two peripheral electrodes with a conducting polymer channel, a continuous electrochemical gradient is produced. With repeated, reverse oxidation and reduction at the two peripheral electrodes, a $1 \mu \mathrm{L}$ water droplet on the center of the channel was moved along the wettability switch for a distance of about $1 \mathrm{~mm}$ [44]. By adding a gate electrode to the channel, an organic electrochemical transistor (OECT) was created in which the steepness of the electrochemical gradient was modulated by the gate potential [47]. Using this device, cell density gradients were produced along the channel of the OECT (Figure 3b, cell density gradient). Further, cell adhesion was tuned and cell attachment was driven towards a narrow area along the channel (Figure 3b, focused cell attachment).

The same mechanism of reversible electrochemical oxidation and reduction can be employed to create conducting polymer films in which ions and solvents can be integrated by reduction, which leads to significant dimensional changes. This phenomenon is called electrochemomechanical deformation and can be used to create soft actuators or transducers for the conversion of electrical power to mechanical work [48] (Figure 4a). When the p-doped conducting polymer is reduced, the doping ion dissociates from the (uncharged) backbone. The free negative charges of the doping ion will be equilibrated by an influx of metal ions and solvents from the surrounding electrolyte. Polyaniline (PANI), polypyrrole (PPy) and polythiophenes (Pth) as conjugated polymer backbones can be used for the fabrication of conducting polymer actuators [49]. Since PANI actuators are operated in acidic environment with $\mathrm{pH}<4$ [50] and Pth actuators reach comparably low strains (\% deformation) in ionic liquid electrolytes [51], PPy actuators are most widely used in biomedical applications [52]. Generally, the dimensional changes in soft actuators made from conducting polymers are the result of ion and solvent influx (Figure 4a, mechanostimulation due to swelling). Thus, the strain in PPy actuators has been shown to depend on the size of ions and solvents exchanged in the oxidation and reduction process. In a biomedical setup, PPy doped with DBS or tosylate are most commonly used to build microactuator systems [53].

An organic electronic microactuator chip for mechanical stimulation of single cells was developed using traditional photolithography and microfabrication techniques. Individually addressable, alternating areas of PPy and SU8 were exposed on a silicon wafer. Renal epithelial cells were seeded on the PPy/SU8 and $\mathrm{Ca}^{2+}$ activity was measured due to mechanical stimulation of cells (Figure 4a, mechanostimulation due to swelling). When the polymer expanded in response to electrochemical switching, an increase of intracellular $\mathrm{Ca}^{2+}$ was observed, accounting for the activation of an autocrine ATP signalling pathway associated with mechanical stimulation of cells [41]. Further, miniature conductive polymer actuators for generation of high pressure in the lab on chip systems were developed by stacking several layers of modified PPy intercalated with electrolyte layers [54]. In a similar approach, PPy/DBS was used as micropump for drug delivery on a multiplexed and switchable microneedle platform [55].

An interesting biomimetic approach is the development of artificial muscle using PPy stretchable actuators. Since the working potential of PPy/DBS films can change as a function of electrolyte concentration, temperature or mechanical stress, it is hypothesized that the material can act not only as an actuator but also as an environmental sensor [56]. Artificial muscles, as electrochemical actuators made up of reactive materials, can therefore respond to the ambient conditions during actuation. In this way, they can be used as actuators, sensing the surrounding conditions during movement [57-59].

Yet another application of a conductive polymer active surface is the controlled release of drugs or biomolecules based on the hypothesis that compounds, entrapped in the polymer during synthesis or fabrication, are released from the polymer matrix due to de-doping and swelling caused by repeated or 
continuous electrochemical switching [60-65] (Figure 4a, drug release due to de-doping/swelling). It has been found that the drug loading capacity of a conductive polymer is not only dependent on the doping level, but can also be increased by introduction of cavities into the material. Thus, de-doping, and the release of the dopant, is probably accompanied by polymer swelling [60,63]. Also, drug release after electrochemical switching of the polymer is not limited to release of negative doping ions when swelling processes are taken into account. This has been demonstrated by precisely tuned delivery of the neutral drug progesterone from a PPy scaffold [65]. Further, networks of PPy nanowires were electrochemically prepared in which the micro- and nano-gaps that separated individual PPy nanowires seem to act as drug storage reservoirs [66]. In this system, drug-loading capacity depends on the volume of the micro- and nano-vacancies, and not the doping level. Hydrophilic and lipophilic drugs can both be loaded into the micro- and nano-gaps due to the amphilicity of the PPy nanowire network [66].

A conductive polymer, in which electrochemical switching leads to complete reorganization, and eventually causes cracking an swelling of the entire polymer film, is the self-doped conductive polymer PEDOT-S:H [67] (Figure 4b, polymer disintegration). The polymer supports a polythiophene backbone structure to which negative charged sulfonate groups are linked via alkyl chains acting like little arms. In its reduced state, the polymer associates with protons to balance its internal negative charge. When an electrical potential is applied and the backbone is oxidized, the protons are expelled and the negatively charged groups on the alkyl arms are drawn to the center of the molecule. This results in a major structural change and eventually leads to swelling, cracking and finally disaggregation of the polymer. Electronically triggered disaggregation of PEDOT-S:H was used to detach urinary bladder epithelial cells from their substrate while conserving important surface antigens as compared to enzymatic detachment [67] (Figure 4b, cell detachment).

\section{Sensors}

Owing to the rough surface topography caused by the polymeric structure, conductive polymers possess a large active surface area compared to metal electrodes of a similar size. The active surface area can be even further increased by fabrication of conductive polymer coated $3 \mathrm{D}$ scaffolds [68-71]. This is facilitated by simple fabrication techniques like electrodeposition or vapor phase deposition. A significantly larger electrode area can potentially yield better signal-to-noise ratios, enhanced sensitivity, and lower detection limits for various sensing applications. Large surface areas are beneficial for label-free sensing, but can also contribute to increase the amount of immobilized biomarker/enzyme in various biosensing applications [72].

Well-known concepts for the biomedical application of PPy based biosensors are the "bioelectronic nose" and "bioelectronic tongue" platforms. For the fabrication of high-performance bioelectronic noses, human olfactory receptor conjugated PPy nanotubes were integrated into a field-effect transistor sensor platform [73]. Binding of the odorant to the olfactory receptor was translated into a detectable signal specific for the respective odorant. Recently, ultrasensitive carbon nanotube and graphene based electronic noses were developed [74-76]. Further, a bioelectronic tongue platform was presented based on a field effect transistor functionalized with human taste receptors [77]. Artificial nose and tongue platforms are mainly directed for use in the food industry to detect spoiled or contaminated products in an early stage during the processing. 
Sensors of neurotransmitters are developed for medical applications. Dopamine sensing in the brain is used to investigate the effects of different treatment options available for Parkinson's disease, which is a neurodegenerative disease linked to dopamine depletion in the striatum [78]. Although dopamine can be detected by positron emission tomography, immunostaining and microdialysis, only electrochemical detection can be applied for continuous monitoring with high temporal resolution [79-82]. Interfering species with oxidization potentials similar to that of dopamine and high impedance of common metal microelectrodes are problematic for the common method of amperometric detection. An approach to specifically detect dopamine in the striatum of the rat in vivo using an implantable microelectrode sensor was presented using the "molecularly imprinted polymer" technique [83]. PPy and dopamine were electrodeposited on a platinum substrate and dopamine was washed out using ethanol, leaving a specifically sized cavity in the polymer film. Through polymer oxidization, carbonyl and carboxylic groups supposedly increased dopamine specificity by attracting its $\mathrm{NH}_{3}$ group and repelling negatively charged molecules. The OPPy-MIP (overoxidized polypyrrole-molecularly imprinted polymer) microelectrodes proved to be superior compared to non-imprinted polymer electrodes in terms of sensitivity and specificity in vitro as well as in vivo [83].

A dopamine biosensor for the detection of dopamine in urine samples was developed by electrodeposition of multi-walled carbon nanotubes, PPy and laccase on a platinum electrode. Detection was carried out by pulse voltammetry and high sensitivity was achieved by chemical oxidation of the phenolic substance by the laccase enzyme on top of electrochemical oxidation [84]. A common feature of PPy sensing applications is the utilization of nanostructures like nanotubes and nanoparticles to increase the effective surface area. Also, enzymes, DNA or other biomolecules are used as dopants in order to increase sensor specificity [85-88]. Exploiting these resources, PPy has been used extensively for sensing of small metabolites like glucose, ascorbic acid and ammonia [89-91] but also for protein detection like haemoglobin [92] and prion protein [93].

Label free sensors based on PEDOT:PSS were developed to monitor the integrity of mucosal linings, formed by epithelial cells in airways, the gastrointestinal tract and the urinary tract. Using an OECT, a constant potential was applied between the drain and source electrodes. Pulses of positive gate potential were applied and the current response was measured. The epithelial integrity was established from the current transient of the gate current (Figure 3b, epithelial integrity sensor) [94]. For a similar approach, a planar, transparent two-electrode sensor was developed for simultaneous electronic and optical monitoring of epithelial monolayer formation and disruption (Figure 3a, epithelial integrity sensor). The phase angle of the current response towards a low sinusoidal $0.1 \mathrm{~V}$ potential was determined for sinusoidal frequencies between 0.1 and $10^{5} \mathrm{~Hz}$. From the recorded phase angle spectrum (PAS), a peak in phase angle amplitude at $10^{3}-10^{5} \mathrm{~Hz}$ was identified, which corresponded to increased cell capacitance as the epithelial monolayer formed on the PEDOT:PSS electrodes. Transparency of the electrode material facilitated immunofluorescence-based identification of the tight junction protein ZO1, demonstrating that a polarized epithelial monolayer had formed. Addition of an ionophore to the polarized monolayer demonstrated that PAS technology is a valuable tool to also monitor disruption of the barrier function. High-resolution data on the kinetics disruptive processes at the polarized epithelium was obtained by quantification of the PAS peak amplitude, with measurements recorded in the partial spectral range every $30 \mathrm{~s}$ [95]. 


\section{Biomedical Applications of Organic Bioelectronics}

\subsection{Neuroscience}

Electrochemistry has been a cornerstone of neuroscience research for decades. Not surprisingly, when the properties of conducting polymers and devices built from conducting polymers were realized, neuroscience was one of the first fields to take advantage of the new breakthroughs. Neuronal cell cultures were among the first experiments to be performed with conducting polymers.

The specific morphology of neuronal cells with dendrites, cell bodies and axons presents specific challenges to common cell culture systems. Novel substrates for neuronal cell culture made from conductive polymers have shown to promote neuronal morphology. Nano-fiber scaffolds made from electrospun poly(ethyleneterephthalate) coated with PEDOT:tosylate promoted multidirectional neurite outgrowth in neuron-like SH-SY5Y cells [68]. Wetspun PPy doped with p-toluene sulfonate has also been used to control and promote rapid and direction growth of nerve cells [96]. Dorsal root ganglia displayed both axonal and Schwann cell alignment along the PPy fibers.

A further advantage of conductive polymers is that they can be used to exert electrical stimulation while supporting neuronal morphology. Thus, in both of the above publications, electrical stimulation was used. It was shown that electrical stimulation induced an intracellular $\mathrm{Ca}^{2+}$ response in the neuronal cell. This is known to lead to activation of multiple signaling cascades related to growth and differentiation $[97,98]$. Furthermore, it could be shown directly that electrical stimulation contributed to accelerated axonal growth [96].

A crucial problem in the focus of today's neuroscience research is to find a way to promote growth of neuronal cells while suppressing growth of glial cells on a surface. This relates to the problems of glial sheathing, which is observed on deep brain stimulation electrodes within two weeks of implantation $[99,100]$. Using PPy, it was shown that by changing the doping agent it was possible to select for the growth of different cell types. PPy doped with fibronectin fragments supported higher densities of glial cells, and PPy doped with nonapeptide CDPGYIGSR supported higher densities of neuroblastoma cells [101]. On the other hand, the doping agent can promote neuronal growth and differentiation of precursor cells into a neuronal phenotype. PPy doped with two different laminin fragments (p31 \& p20) was shown to affect neuronal adhesion and morphology in vitro. Both p31 and p20 doped PPy showed a higher neuronal density than the control surfaces and p20 doped PPy supported significantly longer neurites. Astrocyte adhesion was also significantly higher on both p31 and p20 doped PPy than on the control [102]. Neuronal growth factor (NGF) was used to dope both PPy and PEDOT and was shown to be active from within the polymer using the attachment and neurite development of PC-12 cell as a marker [103].

Exposure to neurotrophic factors or electrical stimulation via conducting polymers can have beneficial effects on neuronal differentiation and morphology. Their combined effect was investigated by measuring outgrowth of neurites from cochlear explants positioned on functionalized PPy [104]. Incorporation of neurotrophin 3 (NT-3) and brain-derived neurotrophic factor (BDNF) into PPy was performed by electrodeposition, yielding up to $244 \pm 7 \mathrm{ng} \mathrm{cm}^{-2}$ BDNF + NT-3. Electrical addressing of the surfaces using biphasic $100 \mu$ s pulses of $\pm 1 \mathrm{~mA} \mathrm{~cm}{ }^{-2}$ facilitated release of BDNF from PPy containing NT-3. While BDNF was released from the unstimulated polymer in form of sustained release, release of NT-3 depended on electrical stimulation of the polymer. Collectively, this showed that neurite 
outgrowth from cochlear explants increased dramatically when neurotrophic factors were released at the same time as electrical stimulation was applied to the polymer.

Use of conductive polymers as electrodes for tissue stimulation has the striking advantage that they can be synthesized in situ. Potentially hazardous surgeries for electrode implantation associated with tissue scarring and gliosis may thus be prevented. Polymerization of PEDOT around neuronal cells was performed by Richardson-Burns et al. [105]. When PEDOT was electrodeposited in a dish containing a living neuronal cell culture, the conductive polymer wrapped closely around the cells and their extensions, sometimes engulfing the cell body. Exposure to electrical current during the electrochemical polymerization process led to permeabilization of the cells' plasma membrane. The researchers used this technology to produce cell-templated PEDOT coatings. Neuron-like cells grown on these cell-templated structures seemed to re-populate the cell-shaped holes, but did not settle into the exact same position as the original cells used for templating [105].

Next, it was demonstrated that a nano- and microscale conductive polymer network could be grown from implanted metal electrodes using in vivo electropolymerization of PEDOT in mouse brain slices. The PEDOT nanofiber network contributed to a large increase in effective surface area of the electrode as compared to that of the bare electrode, as evidenced by decreased impedance and increased charge capacity [106].

An increasingly recognized aspect of conducting polymers in neuroscience is their potential for brain computer interface applications [2,107]. Their ability to translate electronic signals into ionic currents has led to the development of an organic electronic biomimetic neuron (OEBN) that mimics chemical-to-electrical-to-chemical signal transmission of neuronal cells [37] (Figure 2).

\subsection{Infection}

Infections are often caused by bacteria growing in a specific state called biofilm that forms when free-living bacteria attach to surface and transition into a biofilm phenotype. In biofilms, dense aggregates of bacteria are embedded in large amounts of extracellular matrix, which help them to firmly adhere to surfaces $[108,109]$. This feature of the biofilm hides the immunogenic bacterial cells from the immune system and prevents them from being cleared. Biofilm formation is also associated with the development of "persisters," which are cells within a bacterial population with a much slower metabolism than normal. This undermines the mode of action of most antibiotics that need replicating bacteria in order have an effect [110]. In the hospital setting, biofilms are especially associated with implanted medical devices. Invasive surgeries in combination with implantation of prosthesis or a device present both an entry point for the invasion of bacteria and a surface upon which attachment and biofilm formation can be initiated. Treatment of biofilm-associated infections is complicated, and usually, high systemic concentrations of antibiotics administered over a long period of time are required in order to reach sufficiently high concentrations at the infection site. Even then, biofilms reduce biocidal activity and are generally considered irreversible once they are established. Even under aggressive antibiotic administration, surgical intervention can often be the only solution to effectively remove a biofilm [111].

Current research focuses on creating new surfaces for incorporation into medical devices, which could either reduce the chance of biofilm formation or remove biofilm by facilitating more effective bacterial killing. A common way of inducing more effective bacterial killing is to enable the release of 
bactericidal drugs directly from the surface. A benefit of this method is that high concentrations of antibiotic are delivered to infection sites without the need for invasive surgery and high systemic doses of antibiotics (as is the case with oral or intravenous drug delivery) [111]. Releasing antibiotics from a conducting polymer surface as shown previously [61,112] could alleviate this problem, as locally released concentrations are high while systemic concentrations are low.

A preventative measure to avoid biofilm formation on implants might be to promote implant ingrowth and prevent inflammatory processes at the implantation site. To address this, electronically controlled drug release has been demonstrated from PPy films coated on implants and prostheses. Functionalized prostheses fabricated with an antibiotic coating prevented infection at the site of implantation and improved integration of the prosthesis with surrounding tissue [113]. Ti is the most widely used material for bone and joint prostheses in hospitals with a high probability of an infection due to the invasive surgery needed for implantation. Inflammatory events at the implant-bone interface inhibit ingrowth and result in implant failure [114]. Release of anti-inflammatory dexamethasone as well as the antibiotics penicillin and streptomycin was examined from nanoporous PPy films deposited on Ti [112,115]. It was demonstrated that $80 \%$ of drugs were released on demand within 5 cyclic voltammetry sweeps [115]. Furthermore, they showed that while fibroblast adhesion on a PPy film was inhibited, bone-forming osteoblast adhesion was improved on PPy doped with dexamethasone (a common anti-inflammatory).

While treatment or prevention of infections by local drug release can be one approach to handle biofilm formation, another could be early detection by sensing subtle changes in cellular density. The mammalian host is separated from the external environment by specialized epithelial cells creating an epithelial barrier [116-118]. The epithelium is also the main regulator of homeostasis within the body, which also makes it a vulnerable entry point for pathogens. Epithelial barrier function is determined by plasma membrane integrity and the presence of tight junction between cells. During infection, bacteria attempt to circumvent these barriers and are counteracted by the host, which acts to clear the infection and re-establish homeostasis with minimal tissue damage. Tissue microbiology studies [119-121], exploring interactions between invading bacteria and the host have shown intricate effects of bacteria-host interactions involving colonization, epithelial blebbing and tissue ischemia [122,123].

To address the complex questions posed by biology, innovative in vitro models using organic bioelectronics are under development. Conductive polymer based sensors detecting epithelial barrier integrity are an example of such a model. An OECT based sensor has been developed initially dependent on a transwell system, but later advanced towards a planar setup, constructed only from conductive polymers [94,124-127] (Figure 3b, epithelial integrity sensor). Further, a transparent two-electrode PEDOT:PSS sensor was recently introduced for dynamic monitoring of epithelial barrier formation and disruption [95] (Figure 3a, epithelial integrity sensor). Using phase angle spectroscopy, epithelial monolayer formation of renal epithelial cells was monitored for about four days. Further, toxin-induced monolayer disruption was monitored at a high temporal resolution while simultaneous microscopic observation was performed.

Since cell growth, polarity and density are important parameters for all cell culture applications, and not only for the investigation of host-pathogen interactions, continuous electronic sensing could become a new method for optimization of routine cell culture interventions. Using wireless alerts, the researcher could be instantaneously alerted when cells are ready for passage, or if other, more unexpected events occur. Eventually such an automated cell culture system could contribute to early detection of microbial 
contaminations, deterioration and cell de-differentiation. Tissue culture forms the base of personalized regenerative medicine, and the combination of electronic and optical sensing offered by the organic PAS sensor may lead to fully automated systems in many cell biology applications [43].

\subsection{Advanced in vitro Models}

The complexity of the in vivo scenario poses a challenge when building in vitro models aiming to decipher the role of various cell types in tissue homeostasis [121,128]. This has provoked interest in developing improved biomimetic devices and in vitro systems. Early breakthroughs in nanotechnology have demonstrated how physical surface properties such as nanotopography could be used to alter cell behavior. Uroepithelial cells cultured on various nanostructured $\mathrm{TiO}$ surfaces showed varied epithelial cell morphology linked to the differential expression of cytokines and chemokines [129]. Today, the rapidly growing field of organic bioelectronics is providing new opportunities to produce active, patterned surfaces. Organic bioelectronics active surfaces will become helpful in mimicking the dynamic nature of biological processes as their surface properties can be tuned reversibly by application of an electrical potential.

In pyelonephritis (kidney infection), bacterial colonization on the mucosa of the kidney's tubular system causes severe disruption to the tight monolayer of the renal epithelium. Consequently, critical physiological functions such as ion reabsorption from primary urine into the vasculature are damaged. To model this in vitro, patterned organic bioelectronics polymers were employed to produce epithelia that could be actively controlled in a functionalized cell culture dish [42]. As renal epithelial Madin-Darby canine kidney (MDCK) cells were seeded, the active cell culture dish was electronically addressed to produce changes in the redox state of the conductive polymer system PEDOT:tosylate. Thus, MDCK cells were growing well when in contact with the reduced surface, displaying normal cell morphology, proliferation, and tight junction formation, whereas a complete absence or only apoptotic cells were seen on oxidized surfaces (Figure 3a, attachment switch). Furthermore, it was shown that continuous redox switching does not affect cell functionality when attached to the reduced surface because the current flow ceases just a few minutes after switching when the polymer is fully oxidized/reduced $[42,47]$. Different cell types (e.g. fibroblasts) also exhibit tunable attachment properties depending on the redox state of the material. On a microwrinkled conductive polymer interface, guided anisotropic multicellular alignment of murine skeletal muscle was demonstrated on top of a fibroblasts feeder layer. Furthermore, fibroblast attachment and formation of a topographically aligned multicellular layer was not permitted on oxidized PEDOT:PSS layers [130].

A number of mechanisms for cell adhesion and expression of extracellular matrix components make it likely that different cell types are influenced in a variety of ways by the redox state of a conducting polymer. MDCK cells seeded onto the channel of an OECT responded to an electrochemical gradient by attaching at specific positions throughout the channel [47] (Figure 3b, cell density gradient). A gradient of cells was generated and the lowest number of adherent cells was present on the oxidized side. The number of cells gradually increased towards the reduced side, until a sharp drop at the highest reduction potential was observed. Tuning the gate potential and increasing the gradient steepness creates a narrow band of material redox states in which cells are able to proliferate or attach (Figure 3b, focused cell attachment). A linear gradient of mesenchymal fibroblasts and fibroblast cell lines was generated on an OECT channel with PEDOT:tosylate deposited on top of indium tin oxide (ITO) [131]. Thus, it seems 
that redox modulation can be used as a tool to produce 2D patterns that can be applied to multicellular models in vitro. Sequential switching electrochemical surfaces as well as seeding of different cell types in a timely way would allow different and yet functionally complementary cells to be correctly positioned in the same cell culture dish. The systems could be developed into excellent tools to understand intercellular signaling pathways between cells in different tissues.

An increasing number of studies are showing that the 3D distribution of tissues, organs and organ systems can influence local physiology [132,133]. Expanding the idea of planar multicellular 2D cell culture models to 3D tissue models requires methods to release cell layers from surfaces on which they are grown. Stacking cell layers with well-defined 2D structure could ultimately evolve into fully developed 3D tissue or organ models in which communication between cells and cell layers can be examined under more natural conditions. Electro-responsive technologies for the production of cell sheets have been implemented, using the affinity of cells to the RGD peptide as an anchor. In one example, the RGD peptide motif is presented to the cells via an electro-active tether to a self-assembled monolayer (SAM) of alkenethiolates on Au [134]. Applying an electrical potential via the Au surface released fibroblasts evenly over the entire electro-active surface. By reversing this principle with the addition of a cyclopentadiene modified RGD motif, a surface can be modified from a state that prevents to a state that promotes cellular attachment. This mechanism was used to initiate and synchronize fibroblasts migration to specific points on patterned surfaces $[134,135]$. An example of this technology on Au threads has been used to generate capillary-like structures as tissue constructs [136]. Based on the discovery that polyelectrolyte mono- and multilayers desorb with electrochemical polarization of a substrate [137], a polyelectrolyte system deposited onto the conducting polymer ITO was used to release sheets of fibroblasts and osteoblasts after a positive potential was applied to the ITO layer [138]. By applying the previously described polyelectrolyte technology to a micropatterned ITO platform, it was possible to produce micropatterned co-cultures of primary human chondrocytes and human mesenchymal stem cells [139]. Another approach for cell release from a substrate was presented using a self-doped water-soluble conductive polymer [67]. Incorporation of PEDOT-S:H thin-film electrodes into transparent cell culture devices lead to human uroepithelial bladder cells being released upon an electrochemical signal. Cells that were released were viable and functioned normally. Just as importantly, surface antigens were preserved on the cells to a greater extent when this method was used in contrast to traditional trypsination. The PEDOT-S:H system merges high spatial resolution of detachment, green synthesis and electrochemically triggered detachment of cells from surfaces. Automated, local detachment of cells using conductive polymers could be used as informative new tools when studying exfoliation of epithelial cells and wound healing.

\subsection{Cell Biology}

The cellular microenvironment, reactions of cells to external stimuli and interactions of cells with each other are, today, difficult to study on a very small scale. Using the active surfaces or OEIP technology described earlier in this review, cells can be stimulated very locally and in a temporally defined manner without disturbing the cellular microenvironment. Further, using electronic sensors and monitoring cells continuously with high temporal resolution, new dimensions of cellular processes could be uncovered. 
It is, for example, widely accepted that a high blood level of liver-produced c-reactive protein (CRP) is a sign of an infection somewhere in the body [140]. The processes that lead to local activation of CRP at the infection site are, however, widely unknown. Using a biomimetic cell membrane combined with an OEIP Goda et al. [141] created an in vitro environment that replicates the pathogenic progression on a sensing platform to investigate the molecular dynamics of C-reactive protein (CRP). By controlling $\mathrm{pH}$ and $\left[\mathrm{Ca}^{2+}\right]$ in the local microenvironment activation of CRP was monitored in real time and binding kinetics of CPR to the bimimetic membrane were established under defined $\mathrm{pH}$ and $\left[\mathrm{Ca}^{2+}\right]$. At the same time, the process of CRP activation was monitored using TIRF microscopy to show that binding occurred at specific points of the surface where there was a change in either the $\mathrm{pH}$ or $\left[\mathrm{Ca}^{2+}\right]$. Thus, Goda et al. could show that very local changes in $\mathrm{pH}$ and $\left[\mathrm{Ca}^{2+}\right]$ as found at the infection site contribute to CRP activation and local initiation of an immune response.

Another phenomenon difficult to study in cell biology is the effect of mechanical stress and tension on a cell culture. Organs such as the urinary bladder can accommodate large changes in the volume of the liquid. Contraction and expansion of cells in the transitional epithelium allow for the distension required for the bladder to stretch between being empty and full. The transitional epithelium also acts as a barrier, with tight junctions that protect the inside of the body from exposure to the outside. As infection progresses there is a rise in the interstitial pressure caused by an increase in water uptake into the interstitium. Technology that allows the study of mechanotransduction and mechanical stimulation of epithelia in vitro is therefore very important. In tissues and whole organs, mechanotransduction was studied with flexible tissue culture substrates that could be stretched by applying external forces. On the cellular and subcellular level, however, specific technology to apply realistic mechanical stimuli is limited. Svennersten et al. produced an organic electronic microactuator chip for the mechanical stimulation of individual cells [41] (Figure 4a, mechanostimulation due to swelling). Photolithography and microfabrication were used to produce individually addressable, alternating areas of PPy and SU8 on a silicon wafer. Uroepithelial cells were grown on the PPy/SU8 and mechanically stimulated when the polymer expanded due to the electrochemical switching. The increase of intracellular $\left[\mathrm{Ca}^{2+}\right]$ caused the activation of an autocrine ATP signalling pathway that is associated with the mechanical stimulation of cells. Sensitivity of elastic organs to infection whether relaxed or stretched could be studied using this mechanostimulation technology.

Another innovative approach of using organic bioelectronics is as a capture and release system for circulating tumor cells (CTCs) [142,143]. CTCs are cancer cells that break away from the tumor with the capacity to form distant metastases. Detecting CTCs from liquid blood biopsies could aid in the diagnosis of tumor incidence, recurrence and metastases in patients. To achieve a NanoVelcro effect for CTCs, carboxylic acid grafted PEDOT (PEDOTAc) nanostructures were conjugated with an antibody against the epithelial cell adhesion molecule (EpCAM) [142,143]. Highly regular nanorod arrays of PEDOTAc coated with PLL-g-PEG-biotin were conjugated with biotinylated anti-EpCAM via streptavidin. The key concept for the capture and release of the CTCs was the dynamic control of the Pll-g-PEG-biotin coating on the PEDOTAc nanorods. The function of the device was demonstrated by capturing of human breast adenocarcinoma cells, which subsequently could be released by repeated potential sweeping between -0.8 to $0.5 \mathrm{~V}$ [144]. 


\section{Conclusions}

Translation between the "electron" world of electronic devices and the "ion" world of biology is a major challenge in modern medicine. Development of high technology brain-computer interfaces and prostheses can allow paralyzed patients to regain mobility and improve quality of life. Furthermore, cheap and precise sensors will allow for timely medical intervention and facilitate remote medicine to avoid hospitalization. Here, we refer to organic bioelectronics as tools that bring electronic precision into biology and biomedical applications. Advanced in vitro technologies are introduced, which apply active surfaces or ion transport devices to mimic dynamic in vivo environments. We also highlight the potential of ion transport technology to delivery biomolecules with high spatiotemporal resolution and precise electronic control. The numerous practical demonstrations of both the effectiveness of organic bioelectronics and the flexibility of the platform are a good indication that organic bioelectronics will become an important component of the next generation of medical devices, as is shown in the example of the organic electronic biomimetic neuron.

\section{Acknowledgments}

We thank all our partners for their fruitful collaboration in building a highly interdisciplinary research environment. Research in the ARD laboratory is supported by the Swedish Medical Nanoscience Center (www.medicalnanoscience.se), Carl Bennet AB, VINNOVA, Karolinska Institutet, and Familjen Erling-Perssons Stiftelse.

\section{Author Contributions}

The manuscript was written by Susanne Löffler and Benjamin Libberton. Agneta Richter-Dahlfors provided guidance on the organization and preparation of the manuscript and reviewed and streamlined the work. All the authors read and approved the final manuscript.

\section{Conflicts of Interest}

The authors declare no conflict of interest.

\section{References}

1. Berggren, M.; Richter-Dahlfors, A. Organic bioelectronics. Adv. Mater. 2007, 19, 3201-3213.

2. Larsson, K.C.; Kjäll, P.; Richter-Dahlfors, A. Organic bioelectronics for electronic-to-chemical translation in modulation of neuronal signaling and machine-to-brain interfacing. Biochim. Biophys. Acta 2013, 1830, 4334-4344.

3. Owens, R.; Kjäll, P.; Richter-Dahlfors, A.; Cicoira, F. Organic bioelectronics-novel applications in biomedicine. Biochim. Biophys. Acta 2013, 1830, 4283-4285.

4. Richter-Dahlfors, A.; Kjäll, P. Nanotechnologies: Emerging applications in biomedicine. Biochim. Biophys. Acta 2011, 1810, 237-238.

5. Svennersten, K.; Larsson, K.C.; Berggren, M.; Richter-Dahlfors, A. Organic bioelectronics in nanomedicine. Biochim. Biophys. Acta 2011, 1810, 276-285. 
6. Muskovich, M.; Bettinger, C.J. Biomaterials-based electronics: Polymers and interfaces for biology and medicine. Adv. Healthc. Mater. 2012, 1, 248-266.

7. Brédas, J.L.; Heeger, A.J.; Wudl, F. Towards organic polymers with very small intrinsic band gaps. I. Electronic structure of polyisothianaphthene and derivatives. J. Chem. Phys. 1986, 85, 4673-4678.

8. Chiang, C.; Fincher, C.; Park, Y.; Heeger, A.; Shirakawa, H.; Louis, E.; Gau, S.; MacDiarmid, A. Electrical conductivity in doped polyacetylene. Phys. Rev. Lett. 1977, 39, 1098-1101.

9. Heeger, A.J. Semiconducting polymers: The third generation. Chem. Soc. Rev. 2010, 39, 2354-2371.

10. MacDiarmid, A.G.; Mammone, R.J.; Kaner, R.B.; Porter, S.J.; Pethig, R.; Heeger, A.J.; Rosseinsky, D.R. The concept of "doping" of conducting polymers: The role of reduction potentials (and discussion). Philos. Trans. R. Soc. A Math. Phys. Eng. Sci. 1985, 314, 3-15.

11. Chochos, C.L.; Choulis, S.A. How the structural deviations on the backbone of conjugated polymers influence their optoelectronic properties and photovoltaic performance. Progress Polym. Sci. 2011, $36,1326-1414$.

12. Kroon, R.; Lenes, M.; Hummelen, J.C.; Blom, P.W.M.; de Boer, B. Small bandgap polymers for organic solar cells (polymer material development in the last 5 years). Polym. Rev. 2008, 48, 531-582.

13. Roncali, J. Molecular engineering of the band gap of $\pi$-conjugated systems: Facing technological applications. Macromol. Rapid Commun. 2007, 28, 1761-1775.

14. Roncali, J. Synthetic principles for bandgap control in linear pi-conjugated systems. Chem. Rev. 1997, 97, 173-206.

15. Dai, L. Intelligent Macromolecules for Smart Devices: From Materials Synthesis to Device Applications; Springer: London, England, 2004; p. 496.

16. Park, H.-S.; Ko, S.-J.; Park, J.-S.; Kim, J.Y.; Song, H.-K. Redox-active charge carriers of conducting polymers as a tuner of conductivity and its potential window. Sci. Rep. 2013, 3, 2454.

17. Stavrinidou, E.; Leleux, P.; Rajaona, H.; Khodagholy, D.; Rivnay, J.; Lindau, M.; Sanaur, S.; Malliaras, G.G. Direct measurement of ion mobility in a conducting polymer. Adv. Mater. 2013, $25,4488-4493$.

18. Chun, H.; Chung, T.D. Iontronics. Ann. Rev. Anal. Chem. 2015, 8, 441-462.

19. Dhote, V.; Bhatnagar, P.; Mishra, P.K.; Mahajan, S.C.; Mishra, D.K. Iontophoresis: A potential emergence of a transdermal drug delivery system. Sci. Pharm. 2012, 80, 1-28.

20. Kalia, Y.N.; Naik, A.; Garrison, J.; Guy, R.H. Iontophoretic drug delivery. Adv. Drug Deliv. Rev. 2004, 56, 619-658.

21. Li, L.C.; Scudds, R.A. Iontophoresis: An overview of the mechanisms and clinical application. Arthritis Care Res. 1995, 8, 51-61.

22. Isaksson, J.; Kjäll, P.; Nilsson, D.; Robinson, N.D.; Berggren, M.; Richter-Dahlfors, A. Electronic control of $\mathrm{Ca} 2+$ signalling in neuronal cells using an organic electronic ion pump. Nat. Mater. 2007, 6, 673-679.

23. Isaksson, J.; Nilsson, D.; Kjäll, P.; Robinson, N.D.; Richter-Dahlfors, A.; Berggren, M. Electronically controlled ph gradients and proton oscillations. Org. Electron. 2008, 9, 303-309.

24. Tybrandt, K.; Larsson, K.C.; Kurup, S.; Simon, D.T.; Kjäll, P.; Isaksson, J.; Sandberg, M.; Jager, E.W.H.; Richter-Dahlfors, A.; Berggren, M. Translating electronic currents to precise acetylcholine induced neuronal signaling using an organic electrophoretic delivery device. Adv. Mater. 2009, 21, 4442-4446. 
25. Tybrandt, K.; Gabrielsson, E.O.; Berggren, M. Toward complementary ionic circuits: The npn ion bipolar junction transistor. J. Am. Chem. Soc. 2011, 133, 10141-10145.

26. Tybrandt, K.; Larsson, K.C.; Richter-Dahlfors, A.; Berggren, M. Ion bipolar junction transistors. Proc. Natl. Acad. Sci. USA 2010, 107, 9929-9932.

27. Volkov, A.V.; Tybrandt, K.; Berggren, M.; Zozoulenko, I.V. Modeling of charge transport in ion bipolar junction transistors. Langmuir 2014, 30, 6999-7005.

28. Simon, D.T.; Kurup, S.; Larsson, K.C.; Hori, R.; Tybrandt, K.; Goiny, M.; Jager, E.W.H.; Berggren, M.; Canlon, B.; Richter-Dahlfors, A. Organic electronics for precise delivery of neurotransmitters to modulate mammalian sensory function. Nat. Mater. 2009, 8, 742-746.

29. Surwade, S.P.; Chai, S.H.; Choi, J.P.; Wang, X.; Lee, J.S.; Vlassiouk, I.V.; Mahurin, S.M.; Dai, S. Electrochemical control of ion transport through a mesoporous carbon membrane. Langmuir 2014, 30, 3606-3611.

30. Li, D.; Jing, W.; Li, S.; Shen, H.; Xing, W. Electric field-controlled ion transport in tio2 nanochannel. ACS Appl. Mater. Interfaces 2015, 7, 11294-11300.

31. Johnson, M.D.; Miocinovic, S.; McIntyre, C.C.; Vitek, J.L. Mechanisms and targets of deep brain stimulation in movement disorders. Neurotherapeutics 2008, 5, 294-308.

32. Osorio, I.; Frei, M.G.; Manly, B.F.; Sunderam, S.; Bhavaraju, N.C.; Wilkinson, S.B. An introduction to contingent (closed-loop) brain electrical stimulation for seizure blockage, to ultra-short-term clinical trials, and to multidimensional statistical analysis of therapeutic efficacy. J. Clin. Neurophysiol. 2001, 18, 533-544.

33. Georgopoulos, A.P.; Schwartz, A.B.; Kettner, R.E. Neuronal population coding of movement direction. Science 1986, 233, 1416-1419.

34. Hochberg, L.R.; Serruya, M.D.; Friehs, G.M.; Mukand, J.A.; Saleh, M.; Caplan, A.H.; Branner, A.; Chen, D.; Penn, R.D.; Donoghue, J.P. Neuronal ensemble control of prosthetic devices by a human with tetraplegia. Nature 2006, 442, 164-171.

35. Georgopoulos, A.P.; Kettner, R.E.; Schwartz, A.B. Primate motor cortex and free arm movements to visual targets in three-dimensional space. II. Coding of the direction of movement by a neuronal population. J. Neurosci. 1988, 8, 2928-2937.

36. Taylor, D.M.; Tillery, S.I.; Schwartz, A.B. Direct cortical control of 3d neuroprosthetic devices. Science 2002, 296, 1829-1832.

37. Simon, D.T.; Larsson, K.C.; Nilsson, D.; Burstrom, G.; Galter, D.; Berggren, M.; Richter-Dahlfors, A. An organic electronic biomimetic neuron enables auto-regulated neuromodulation. Biosens. Bioelectron. 2015, 71, 359-364.

38. Jeong, J.W.; McCall, J.G.; Zhang, Y.; Huang, Y.; Bruchas, M.R.; Rogers, J.A. Soft microfluidic neural probes for wireless drug delivery in freely behaving mice, In Proceedings of the International Conference on Solid-State Sensors, Actuators and Microsystems, Anchorage, AK, USA, 21-25 June 2015; pp. 2264-2267.

39. Pernaut, J.-M.; Reynolds, J.R. Use of conducting electroactive polymers for drug delivery and sensing of bioactive molecules. A redox chemistry approach. J. Phys. Chem. B 2000, 104, 4080-4090.

40. Balint, R.; Cassidy, N.J.; Cartmell, S.H. Conductive polymers: Towards a smart biomaterial for tissue engineering. Acta Biomater. 2014, 10, 2341-2353. 
41. Svennersten, K.; Berggren, M.; Richter-Dahlfors, A.; Jager, E.W.H. Mechanical stimulation of epithelial cells using polypyrrole microactuators. Lab Chip 2011, 11, 3287-3293.

42. Svennersten, K.; Bolin, M.H.; Jager, E.W.H.; Berggren, M.; Richter-Dahlfors, A. Electrochemical modulation of epithelia formation using conducting polymers. Biomaterials 2009, 30, 6257-6264.

43. Löffler, S.; Libberton, B.; Richter-Dahlfors, A. Organic bioelectronics in infection. J. Mater. Chem. B 2015, 3, 4979-4992.

44. Isaksson, J.; Tengstedt, C.; Fahlman, M.; Robinson, N.; Berggren, M. A solid-state organic electronic wettability switch. Adv. Mater. 2004, 16, 316-320.

45. Liu, M.; Nie, F.Q.; Wei, Z.; Song, Y.; Jiang, L. In situ electrochemical switching of wetting state of oil droplet on conducting polymer films. Langmuir 2010, 26, 3993-3997.

46. Chang, J.H.; Hunter, I.W. Characterization and control of the wettability of conducting polymer thin films. In Materials Research Society Symposium Proceedings; Materials Research Society: Cambridge, England, 2009.

47. Bolin, M.; Svennersten, K.; Nilsson, D.; Sawatdee, A.; Jager, E.W.H.; Richter-Dahlfors, A.; Berggren, M. Active control of epithelial cell density gradients grown along the channel of an organic electrochemical transistor. Adv. Mater. 2009, 21, 4379-4382.

48. Keiichi, K.; Hisashi, F.; Masakatsu, K.; Wataru, T. Conducting polymer soft actuators based on polypyrrole films - energy conversion efficiency. Smart Mater. Struct. 2007, 16, S250.

49. Kaneto, K.; Somekawa, H.; Takashima, W. Soft actuators based on conducting polymers: Recent progress. SPIE Proc. 2003, 5051, doi:10.1117/12.484716.

50. Kaneto, K.; Nakashima, M.; Takashima, W. Improvement of electrochemical deformation of conducting polymers: Strain, force, and response. SPIE Proc. 2004, 5385, doi:10.1117/12.536654.

51. Pattavarakorn, D.; Youngta, P.; Jaesrichai, S.; Thongbor, S.; Chaimongkol, P. Electroactive performances of conductive polythiophene/hydrogel hybrid artificial muscle. Energy Procedia 2013, 34, 673-681.

52. Madden, J.D. Polypyrrole actuators: Properties and initial applications. In Electroactive Polymers for Robotic Applications; Kim, K., Tadokoro, S., Eds.; Springer: London, UK, 2007; pp. 121-152.

53. Wang, X.; Smela, E. Color and volume change in PPy(DBS). J. Phys. Chem. C 2009, 113, 359-368.

54. Hiraoka, M.; Fiorini, P.; O’Callaghan, J.; Yamashita, I.; van Hoof, C.; Op de Beeck, M. Miniature conductive polymer actuators for high pressure generation in lab on chip systems. Sens. Actuators A Phys. 2012,177, 23-29.

55. Valdes-Ramirez, G.; Windmiller, J.R.; Claussen, J.C.; Martinez, A.G.; Kuralay, F.; Zhou, M.; Zhou, N.; Polsky, R.; Miller, P.R.; Narayan, R.; et al. Multiplexed and switchable release of distinct fluids from microneedle platforms via conducting polymer nanoactuators for potential drug delivery. Sens. Actuators B Chem. 2012, 161, 1018-1024.

56. Conzuelo, L.V.; Arias-Pardilla, J.; Cauich-Rodriguez, J.V.; Smit, M.A.; Otero, T.F. Sensing and tactile artificial muscles from reactive materials. Sensors 2010, 10, 2638-2674.

57. Otero, T.F.; Martinez, J.G. Structural electrochemistry: Conductivities and ionic content from rising reduced polypyrrole films. Adv. Funct. Mater. 2014, 24, 1259-1264.

58. Schroeder, P.; Schotter, J.; Shoshi, A.; Eggeling, M.; Bethge, O.; Hutten, A.; Bruck1, H. Artificial cilia of magnetically tagged polymer nanowires for biomimetic mechanosensing. Bioinspiration Biomimicry 2011, 6, 046007. 
59. Ismail, Y.A.; Martínez, J.G.; Al Harrasi, A.S.; Kim, S.J.; Otero, T.F. Sensing characteristics of a conducting polymer/hydrogel hybrid microfiber artificial muscle. Sens. Actuators B Chem. 2011, 160, 1180-1190.

60. Abidian, M.R.; Kim, D.H.; Martin, D.C. Conducting-polymer nanotubes for controlled drug release. Adv. Mater. 2006, 18, 405-409.

61. Esrafilzadeh, D.; Razal, J.M.; Moulton, S.E.; Stewart, E.M.; Wallace, G.G. Multifunctional conducting fibres with electrically controlled release of ciprofloxacin. J. Control. Release 2013, 169, 313-320.

62. Poole-Warren, L.; Goding, J. Challenges of therapeutic delivery using conducting polymers. Ther. Deliv. 2012, 3, 421-427.

63. Sharma, M.; Waterhouse, G.I.N.; Loader, S.W.C.; Garg, S.; Svirskis, D. High surface area polypyrrole scaffolds for tunable drug delivery. Int. J. Pharm. 2013, 443, 163-168.

64. Svirskis, D.; Travas-Sejdic, J.; Rodgers, A.; Garg, S. Electrochemically controlled drug delivery based on intrinsically conducting polymers. J. Control. Release 2010, 146, 6-15.

65. Svirskis, D.; Sharma, M.; Yu, Y.; Garg, S. Electrically switchable polypyrrole film for the tunable release of progesterone. Ther. Deliv. 2013, 4, 307-313.

66. Jiang, S.; Sun, Y.; Cui, X.; Huang, X.; He, Y.; Ji, S.; Shi, W.; Ge, D. Enhanced drug loading capacity of polypyrrole nanowire network for controlled drug release. Synth. Metals 2013, 163, 19-23.

67. Persson, K.M.; Karlsson, R.; Svennersten, K.; Löffler, S.; Jager, E.W.H.; Richter-Dahlfors, A.; Konradsson, P.; Berggren, M. Electronic control of cell detachment using a self-doped conducting polymer. Adv. Mater. 2011, 23, 4403-4408.

68. Bolin, M.; Svennersten, K.; Wang, X.; Chronakis, I.S.; Richter-Dahlfors, A.; Jager, E.; Berggren, M. Nano-fiber scaffold electrodes based on pedot for cell stimulation. Sens. Actuators 2009, 142, 451-456.

69. Hardy, J.G.; Lee, J.Y.; Schmidt, C.E. Biomimetic conducting polymer-based tissue scaffolds. Curr. Opin. Biotechnol. 2013, 24, 847-854.

70. Shahini, A.; Yazdimamaghani, M.; Walker, K.J.; Eastman, M.A.; Hatami-Marbini, H.; Smith, B.J.; Ricci, J.L.; Madihally, S.V.; Vashaee, D.; Tayebi, L. 3d conductive nanocomposite scaffold for bone tissue engineering. Int. J. Nanomed. 2014, 9, 167-181.

71. Wan, A.M.-D.; Inal, S.; Williams, T.; Wang, K.; Leleux, P.; Estevez, L.; Giannelis, E.P.; Fischbach, C.; Malliaras, G.G.; Gourdon, D. 3d conducting polymer platforms for electrical control of protein conformation and cellular functions. J. Mater. Chem. B 2015, 3, 5040-5048.

72. Summerlot, D.; Kumar, A.; Das, S.; Goldstein, L.; Seal, S.; Diaz, D.; Cho, H.J. Nanoporous gold electrode for electrochemical sensors in biological environment. Procedia Eng. 2011, 25, 1457-1460.

73. Yoon, H.; Lee, S.H.; Kwon, O.S.; Song, H.S.; Oh, E.H.; Park, T.H.; Jang, J. Polypyrrole nanotubes conjugated with human olfactory receptors: High-performance transducers for fet-type bioelectronic noses. Angew. Chem. Int. Ed. 2009, 48, 2755-2758.

74. Dian, J.; Konečný, M.; Broncová, G.; Krond’ák, M.; Matolínová, I. Electrochemical fabrication and characterization of porous silicon/polypyrrole composites and chemical sensing of organic vapors. Int. J. Electrochem. Sci. 2013, 8, 1559-1572.

75. Lee, S.H.; Kwon, O.S.; Song, H.S.; Park, S.J.; Sung, J.H.; Jang, J.; Park, T.H. Mimicking the human smell sensing mechanism with an artificial nose platform. Biomaterials 2012, 33, 1722-1729. 
76. Park, S.J.; Kwon, O.S.; Lee, S.H.; Song, H.S.; Park, T.H.; Jang, J. Ultrasensitive flexible graphene based field-effect transistor (FET)-type bioelectronic nose. Nano Lett. 2012, 12, 5082-5090.

77. Song, H.S.; Kwon, O.S.; Lee, S.H.; Park, S.J.; Kim, U.K.; Jang, J.; Park, T.H. Human taste receptor-functionalized field effect transistor as a human-like nanobioelectronic tongue. Nano Lett. 2013, 13, 172-178.

78. Beitz, J.M. Parkinson's disease: A review. Front. Biosci. 2014, 6, 65-74.

79. Chen, Y.C.; Galpern, W.R.; Brownell, A.L.; Matthews, R.T.; Bogdanov, M.; Isacson, O.; Keltner, J.R.; Beal, M.F.; Rosen, B.R.; Jenkins, B.G. Detection of dopaminergic neurotransmitter activity using pharmacologic MRI: Correlation with PET, microdialysis, and behavioral data. Magn. Reson. Med. 1997, 38, 389-398.

80. Kim, Y.-R.; Bong, S.; Kang, Y.-J.; Yang, Y.; Mahajan, R.K.; Kim, J.S.; Kim, H. Electrochemical detection of dopamine in the presence of ascorbic acid using graphene modified electrodes. Biosens. Bioelectron. 2010, 25, 2366-2369.

81. Robinson, D.L.; Venton, B.J.; Heien, M.L.A.V.; Wightman, R.M. Detecting subsecond dopamine release with fast-scan cyclic voltammetry in vivo. Clin. Chem. 2003, 49, 1763-1773.

82. Sansuk, S.; Bitziou, E.; Joseph, M.B.; Covington, J.A.; Boutelle, M.G.; Unwin, P.R.; Macpherson, J.V. Ultrasensitive detection of dopamine using a carbon nanotube network microfluidic flow electrode. Anal. Chem. 2013, 85, 163-169.

83. Tsai, T.-C.; Han, H.-Z.; Cheng, C.-C.; Chen, L.-C.; Chang, H.-C.; Chen, J.-J.J. Modification of platinum microelectrode with molecularly imprinted over-oxidized polypyrrole for dopamine measurement in rat striatum. Sens. Actuators B Chem. 2012, 171-172, 93-101.

84. Cesarino, I.; Galesco, H.V.; Moraes, F.C.; Lanza, M.R.V.; Machado, S.A.S. Biosensor based on electrocodeposition of carbon nanotubes/polypyrrole/laccase for neurotransmitter detection. Electroanalysis 2013, 25, 394-400.

85. Daniel, S.; Rao, T.P.; Rao, K.S.; Rani, S.U.; Naidu, G.R.K.; Lee, H.-Y.; Kawai, T. A review of DNA functionalized/grafted carbon nanotubes and their characterization. Sens. Actuators B Chem. 2007, 122, 672-682.

86. Putzbach, W.; Ronkainen, N. Immobilization techniques in the fabrication of nanomaterial-based electrochemical biosensors: A review. Sensors 2013, 13, 4811-4840.

87. Wang, J. Carbon-nanotube based electrochemical biosensors: A review. Electroanalysis 2005, 17, $7-14$.

88. Huang, J.; Liu, Y.; You, T. Carbon nanofiber based electrochemical biosensors: A review. Anal. Methods 2010, 2, 202-211.

89. Yuqing, M.; Jianrong, C.; Xiaohua, W. Using electropolymerized non-conducting polymers to develop enzyme amperometric biosensors. Trends Biotechnol. 2004, 22, 227-231.

90. Harper, A.; Anderson, M.R. Electrochemical glucose sensors-developments using electrostatic assembly and carbon nanotubes for biosensor construction. Sensors 2010, 10, 8248-8274.

91. Rahman, M.; Kumar, P.; Park, D.-S.; Shim, Y.-B. Electrochemical sensors based on organic conjugated polymers. Sensors 2008, 8, 118-141.

92. Chawla, S.; Pundir, C.S. An amperometric hemoglobin a1c biosensor based on immobilization of fructosyl amino acid oxidase onto zinc oxide nanoparticles-polypyrrole film. Anal. Biochem. 2012, $430,156-162$. 
93. Miodek, A.; Poturnayova, A.; Snejdarkova, M.; Hianik, T.; Korri-Youssoufi, H. Binding kinetics of human cellular prion detection by DNA aptamers immobilized on a conducting polypyrrole. Anal. Bioanal. Chem. 2013, 405, 2505-2514.

94. Ramuz, M.; Hama, A.; Huerta, M.; Rivnay, J.; Leleux, P.; Owens, R.M. Combined optical and electronic sensing of epithelial cells using planar organic transistors. Adv. Mater. 2014, 26, 7083-7090.

95. Löffler, S.; Richter-Dahlfors, A. Phase angle spectroscopy on transparent conducting polymer electrodes for real-time measurement of epithelial barrier integrity. J. Mater. Chem. B 2015, 3, 4997-5000.

96. Quigley, A.F.; Razal, J.M.; Thompson, B.C.; Moulton, S.E.; Kita, M.; Kennedy, E.L.; Clark, G.M.; Wallace, G.G.; Kapsa, R.M. A conducting-polymer platform with biodegradable fibers for stimulation and guidance of axonal growth. Adv. Mater. 2009, 21, 4393-4397.

97. McCaig, C.D.; Song, B.; Rajnicek, A.M. Electrical dimensions in cell science. J. Cell Sci. 2009, 122, 4267-4276.

98. Wang, M.; Li, P.; Liu, M.; Song, W.; Wu, Q.; Fan, Y. Potential protective effect of biphasic electrical stimulation against growth factor-deprived apoptosis on olfactory bulb neural progenitor cells through the brain-derived neurotrophic factor-phosphatidylinositol 3'-kinase/akt pathway. Exp. Biol. Med. 2013, 238, 951-959.

99. Cicchetti, F.; Barker, R.A. The glial response to intracerebrally delivered therapies for neurodegenerative disorders: Is this a critical issue? Front. Pharmacol. 2014, 5, doi:10.3389/ fphar.2014.00139.

100. Lempka, S.F.; Miocinovic, S.; Johnson, M.D.; Vitek, J.L.; McIntyre, C.C. In vivo impedance spectroscopy of deep brain stimulation electrodes. J. Neural Eng. 2009, 6, 046001.

101. Cui, X.; Lee, V.A.; Raphael, Y.; Wiler, J.A.; Hetke, J.F.; Anderson, D.J.; Martin, D.C. Surface modification of neural recording electrodes with conducting polymer/biomolecule blends. J. Biomed. Mater. Res. 2001, 56, 261-272.

102. Stauffer, W.R.; Cui, X.T. Polypyrrole doped with 2 peptide sequences from laminin. Biomaterials 2006, 27, 2405-2413.

103. Kim, D.H.; Richardson-Burns, S.M.; Hendricks, J.L.; Sequera, C.; Martin, D.C. Effect of immobilized nerve growth factor on conductive polymers: Electrical properties and cellular response. Adv. Funct. Mater. 2007, 17, 79-86.

104. Thompson, B.C.; Richardson, R.T.; Moulton, S.E.; Evans, A.J.; O’Leary, S.; Clark, G.M.; Wallace, G.G. Conducting polymers, dual neurotrophins and pulsed electrical stimulation-Dramatic effects on neurite outgrowth. J. Control. Release 2010, 141, 161-167.

105. Richardson-Burns, S.M.; Hendricks, J.L.; Foster, B.; Povlich, L.K.; Kim, D.H.; Martin, D.C. Polymerization of the conducting polymer poly(3,4-ethylenedioxythiophene) (PEDOT) around living neural cells. Biomaterials 2007, 28, 1539-1552.

106. Richardson-Burns, S.M.; Hendricks, J.L.; Martin, D.C. Electrochemical polymerization of conducting polymers in living neural tissue. J. Neural Eng. 2007, 4, L6-L13.

107. Baek, H.J.; Kim, H.S.; Heo, J.; Lim, Y.G.; Park, K.S. Brain-computer interfaces using capacitive measurement of visual or auditory steady-state responses. J. Neural Eng. 2013, 10, 024001.

108. Bjarnsholt, T.; Alhede, M.; Alhede, M.; Eickhardt-Sørensen, S.R.; Moser, C.; Kühl, M.; Jensen, P.Ø.; Høiby, N. The in vivo biofilm. Trends Microbiol. 2013, 21, 466-474. 
109. Høiby, N.; Bjarnsholt, T.; Givskov, M.; Molin, S.; Ciofu, O. Antibiotic resistance of bacterial biofilms. Int. J. Antimicrob. Agents 2010, 35, 322-332.

110. Keren, I.; Shah, D.; Spoering, A.; Kaldalu, N.; Lewis, K. Specialized persister cells and the mechanism of multidrug tolerance in E. coli. J. Bacteriol. 2004, 186, 8172-8180.

111. Darouiche, R.O. Treatment of infections associated with surgical implants. N. Engl. J. Med. 2004, 350, 1422-1429.

112. Sirivisoot, S.; Pareta, R.A.; Webster, T.J. A conductive nanostructured polymer electrodeposited on titanium as a controllable, local drug delivery platform. J. Biomed. Mater. Res. Part A 2011, 99, 586-597.

113. Zelikin, A.N. Drug releasing polymer thin films: New era of surface-mediated drug delivery. ACS Nano 2010, 4, 2494-2509.

114. Brunette, D.M.; Tengvall, P.; Textor, M.; Thomsen, P. Titanium in Medicine: Material Science, Surface Science, Engineering, Biological Responses, and Medical Applications; Springer: New York, NY, USA, 2001; pp. 13-24.

115. Sirivisoot, S.; Pareta, R.; Webster, T.J. Electrically controlled drug release from nanostructured polypyrrole coated on titanium. Nanotechnology 2011, 22, 085101.

116. Peterson, L.W.; Artis, D. Intestinal epithelial cells: Regulators of barrier function and immune homeostasis. Nat. Rev. Immunol. 2014, 14, 141-153.

117. Marchiando, A.M.; Graham, W.V.; Turner, J.R. Epithelial barriers in homeostasis and disease. Ann. Rev. Pathol. 2010, 5, 119-144.

118. Guttman, J.A.; Finlay, B.B. Tight junctions as targets of infectious agents. Biochim. Biophys. Acta 2009, 1788, 832-841.

119. Choong, F.X.; Regberg, J.; Udekwu, K.I.; Richter-Dahlfors, A. Intravital models of infection lay the foundation for tissue microbiology. Future Microbiol. 2012, 7, 519-533.

120. Melican, K.; Richter-Dahlfors, A. Real-time live imaging to study bacterial infections in vivo. Curr. Opin. Microbiol. 2009, 12, 31-36.

121. Richter-Dahlfors, A.; Rhen, M.; Udekwu, K. Tissue microbiology provides a coherent picture of infection. Curr. Opin. Microbiol. 2012, 15, 15-22.

122. Månsson, L.E.; Melican, K.; Boekel, J.; Sandoval, R.M.; Hautefort, I.; Tanner, G.A.; Molitoris, B.A.; Richter-Dahlfors, A. Real-time studies of the progression of bacterial infections and immediate tissue responses in live animals. Cell. Microbiol. 2006, 9, 413-424.

123. Melican, K.; Boekel, J.; Månsson, L.E.; Sandoval, R.M.; Tanner, G.A.; Källskog, O.; Palm, F.; Molitoris, B.A.; Richter-Dahlfors, A. Bacterial infection-mediated mucosal signalling induces local renal ischaemia as a defence against sepsis. Cell. Microbiol. 2008, 10, 1987-1998.

124. Jimison, L.H.; Tria, S.A.; Khodagholy, D.; Gurfinkel, M.; Lanzarini, E.; Hama, A.; Malliaras, G.G.; Owens, R.M. Measurement of barrier tissue integrity with an organic electrochemical transistor. Adv. Mater. 2012, 24, 5919-5923.

125. Tria, S.; Jimison, L.H.; Hama, A.; Bongo, M.; Owens, R.M. Sensing of EGTA mediated barrier tissue disruption with an organic transistor. Biosensors 2013, 3, 44-57.

126. Tria, S.A.; Ramuz, M.; Huerta, M.; Leleux, P.; Rivnay, J.; Jimison, L.H.; Hama, A.; Malliaras, G.G.; Owens, R.M. Dynamic monitoring of salmonella typhimurium infection of polarized epithelia using organic transistors. Adv. Healthc. Mater. 2014, 3, 1053-1060. 
127. Tria, S.A.; Ramuz, M.; Jimison, L.H.; Hama, A.; Owens, R.M. Sensing of barrier tissue disruption with an organic electrochemical transistor. J. Vis. Exp. 2014, e51102, doi:10.3791/51102.

128. Van Regenmortel, M.H.V. Reductionism and complexity in molecular biology. Scientists now have the tools to unravel biological and overcome the limitations of reductionism. EMBO Rep. 2004, 5, 1016-1020.

129. Andersson, A.-S.; Bäckhed, F.; von Euler, A.; Richter-Dahlfors, A.; Sutherland, D.; Kasemo, B. Nanoscale features influence epithelial cell morphology and cytokine production. Biomaterials 2003, 24, 3427-3436.

130. Greco, F.; Fujie, T.; Ricotti, L.; Taccola, S.; Mazzolai, B.; Mattoli, V. Microwrinkled conducting polymer interface for anisotropic multicellular alignment. ACS Appl. Mater. Interfaces 2013, 5, 573-584.

131. Plummer, S.T.; Wang, Q.; Bohn, P.W.; Stockton, R.; Schwartz, M.A. Electrochemically derived gradients of the extracellular matrix protein fibronectin on gold. Langmuir 2003, 19, 7528-7536.

132. Melican, K.; Boekel, J.; Ryden-Aulin, M.; Richter-Dahlfors, A. Novel innate immune functions revealed by dynamic, real-time live imaging of bacterial infections. Crit. Rev. Immunol. 2010, 30, 107-117.

133. Boekel, J.; Källskog, O.; Rydén-Aulin, M.; Rhen, M.; Richter-Dahlfors, A. Comparative tissue transcriptomics reveal prompt inter-organ communication in response to local bacterial kidney infection. BMC Genomics 2011, 12, doi:10.1186/1471-2164-12-123.

134. Inaba, R.; Khademhosseini, A.; Suzuki, H.; Fukuda, J. Electrochemical desorption of self-assembled monolayers for engineering cellular tissues. Biomaterials 2009, 30, 3573-3579.

135. Yousaf, M.N.; Houseman, B.T.; Mrksich, M. Turning on cell migration with electroactive substrates. Angew. Chem. Int. Ed. 2001, 40, 1093-1096.

136. Seto, Y.; Inaba, R.; Okuyama, T.; Sassa, F.; Suzuki, H.; Fukuda, J. Engineering of capillary-like structures in tissue constructs by electrochemical detachment of cells. Biomaterials 2010, 31, 2209-2215.

137. Tang, Z.; Wang, Y.; Podsiadlo, P.; Kotov, N.A. Biomedical applications of layer-by-layer assembly: From biomimetics to tissue engineering. Adv. Mater. 2006, 18, 3203-3224.

138. Guillaume-Gentil, O.; Akiyama, Y.; Schuler, M.; Tang, C.; Textor, M.; Yamato, M.; Okano, T.; Vörös, J. Polyelectrolyte coatings with a potential for electronic control and cell sheet engineering. Adv. Mater. 2008, 20, 560-565.

139. Guillaume-Gentil, O.; Gabi, M.; Zenobi-Wong, M.; Vörös, J. Electrochemically switchable platform for the micro-patterning and release of heterotypic cell sheets. Biomed. Microdevices 2011, 13, 221-230.

140. Pepys, M.B.; Hirschfield, G.M. C-reactive protein: A critical update. J. Clin. Investig. 2003, 111, 1805-1812.

141. Goda, T.; Kjall, P.; Ishihara, K.; Richter-Dahlfors, A.; Miyahara, Y. Biomimetic interfaces reveal activation dynamics of c-reactive protein in local microenvironments. Adv. Healthc. Mater. 2014, 3, 1733-1738.

142. Hsiao, Y.S.; Luo, S.C.; Hou, S.; Zhu, B.; Sekine, J.; Kuo, C.W.; Chueh, D.Y.; Yu, H.H.; Tseng, H.R.; Chen, P. $3 \mathrm{~d}$ bioelectronic interface: Capturing circulating tumor cells onto conducting polymer-based micro/nanorod arrays with chemical and topographical control. Small 2014, 10, 3012-3017. 
143. Sekine, J.; Luo, S.C.; Wang, S.; Zhu, B.; Tseng, H.R.; Yu, H.H. Functionalized conducting polymer nanodots for enhanced cell capturing: The synergistic effect of capture agents and nanostructures. Adv. Mater. 2011, 23, 4788-4792.

144. Hsiao, Y.-S.; Ho, B.-C.; Yan, H.-X.; Kuo, C.-W.; Chueh, D.-Y.; Yu, H.-H.; Chen, P. Integrated 3D conducting polymer-based bioelectronics for capture and release of circulating tumor cells. J. Mater. Chem. B 2015, 3, 5103-5110.

(C) 2015 by the authors; licensee MDPI, Basel, Switzerland. This article is an open access article distributed under the terms and conditions of the Creative Commons Attribution license (http://creativecommons.org/licenses/by/4.0/). 